DOI 10.4171/JEMS/394

F. Cellarosi · Ya. G. Sinai

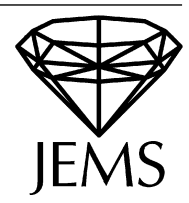

\title{
Ergodic properties of square-free numbers
}

Received March 1, 2012 and in revised form May 24, 2012

Abstract. We construct a natural invariant measure concentrated on the set of square-free numbers, and invariant under the shift. We prove that the corresponding dynamical system is isomorphic to a translation on a compact Abelian group. This implies that this system is not weakly mixing and has zero measure-theoretical entropy.

Keywords. Square-free numbers, correlation functions, dynamical systems with pure point spectrum, ergodicity

\section{Introduction and notations}

Let $\mathcal{P}$ be the set of prime numbers. By $p$ (with or without indices) we will always denote an element of $\mathcal{P}$. A positive integer $n$ is square-free if $p^{2} \nmid n$ for every $p$. Denote the set of all square-free numbers by $\mathcal{Q}$ (for quadratfrei). The indicator of the set $\mathcal{Q}$ is the function $n \mapsto \mu^{2}(n)$, where $\mu$ is the Möbius function:

$$
\mu(n)= \begin{cases}1 & \text { if } n=1, \\ 0 & \text { if } n \text { is not square-free, } \\ (-1)^{k} & \text { if } n \text { is the product of } k \text { distinct primes. }\end{cases}
$$

The functions $\mu$ and $\mu^{2}$ are of great importance in number theory because of their connection with the Riemann zeta function. For example,

$$
\sum_{n=1}^{\infty} \frac{\mu(n)}{n^{s}}=\frac{1}{\zeta(s)}, \quad \sum_{n=1}^{\infty} \frac{\mu^{2}(n)}{n^{s}}=\frac{\zeta(s)}{\zeta(2 s)} .
$$

Furthermore, the estimate $\left|\sum_{n \leq N} \mu(n)\right|=\mathcal{O}_{\varepsilon}\left(N^{1 / 2+\varepsilon}\right)$ as $N \rightarrow \infty$ is equivalent to the Riemann Hypothesis. P. Sarnak [12] has recently addressed a number of statistical and ergodic properties of the sequences $(\mu(n))_{n}$ and $\left(\mu^{2}(n)\right)_{n}$.

F. Cellarosi: Institute for Advanced Study, Princeton, NJ 08540, U.S.A.; e-mail: fcellaro@ias.edu Ya. G. Sinai: Mathematics Department, Princeton University, Princeton, NJ 08540, U.S.A., and Landau Institute of Theoretical Physics, Russian Academy of Sciences, Moscow, Russia; e-mail: sinai@math.princeton.edu

Mathematics Subject Classification (2010): 37A35, 37A45, 28D99 


\subsection{Notations}

We shall use the standard notation $e(x)=e^{2 \pi i x}$. For every integer $n$ denote by $\omega(n)$ the number of its distinct prime factors. For example, $\omega(1)=0$ and $\omega(2 \cdot 3)=\omega\left(2^{10} \cdot 3^{7}\right)=$ $\omega(7 \cdot 23)=2$. We shall also use the notations

$$
\mathcal{P}(n)=\{p: p \mid n\}, \quad \mathcal{P}_{2}(n)=\left\{p: p^{2} \mid n\right\} .
$$

Notice that if $n \in \mathcal{Q}$, then $|\mathcal{P}(n)|=\omega(n), \mathcal{P}_{2}(n)=\emptyset$, and $\mathcal{P}_{2}\left(n^{2}\right)=\mathcal{P}(n)$. For every finite set $\mathcal{A} \subset \mathcal{P}$, define

$$
[\mathcal{A}]=\prod_{p \in \mathcal{A}} p .
$$

In particular $[\emptyset]=1$. Notice that if $\mathcal{A}, \mathcal{B}$ are disjoint, then $[\mathcal{A} \cup \mathcal{B}]=[\mathcal{A}][\mathcal{B}]$ and $[\mathcal{A} \cap \mathcal{B}]=1$.

\section{Formulation of the results}

The goal of this paper is to describe a dynamical system 'naturally' associated to $\mathcal{Q}$ and study its statistical and ergodic properties.

\subsection{Correlation functions}

The first step is the construction of correlation functions for $\mathcal{Q}$. Choose $r$ integers $0 \leq$ $k_{1}<\cdots<k_{r}$ and consider the set

$$
\mathcal{Q}_{N}\left(k_{1}, \ldots, k_{r}\right)=\left\{n \leq N: \mu^{2}(n)=\mu^{2}\left(n+k_{1}\right)=\cdots=\mu^{2}\left(n+k_{r}\right)=1\right\} .
$$

The ratio

$$
\mathbb{E}_{N}\left(k_{1}, \ldots, k_{r}\right):=\left|\mathcal{Q}_{N}\left(k_{1}, \ldots, k_{r}\right)\right| / N
$$

is the frequency of square-free integers $n \leq N$ for which $n+k_{1}, \ldots, n+k_{r}$ are also squarefree. It also gives the expectation (hence the notation $\mathbb{E})$ of the product $\mu^{2}(n) \mu^{2}(n+$ $\left.k_{1}\right) \cdots \mu^{2}\left(n+k_{r}\right)$ with respect to the uniform measure on $\{1, \ldots, N\}$. Notice, by taking $r=1$ and $k_{1}=0$, that $\mathcal{Q}_{N}(0)$ is simply the set of all square-free numbers not greater than $N$. It is well known that

$$
\lim _{N \rightarrow \infty} \mathbb{E}_{N}(0)=6 / \pi^{2} \approx 0.6079271018
$$

We include the proof of (2) and some of its generalizations in Section 2 (see Theorems 2.1-2.3). The study of $\mathbb{E}_{N}\left(k_{1}, \ldots, k_{r}\right)$ as $N \rightarrow \infty$ is also classical: see L. Mirsky [5], R. R. Hall [2], K. M. Tsang [14], D. R. Heath-Brown [4]. It is known that the limits

$$
c_{r+1}\left(k_{1}, \ldots, k_{r}\right)=\lim _{N \rightarrow \infty} \mathbb{E}_{N}\left(k_{1}, \ldots, k_{r}\right)
$$


exist. We shall refer to $c_{r+1}$ as the $(r+1)$-st correlation function for $\mathcal{Q}$. Various formulæ for $c_{r+1}\left(k_{1}, \ldots, k_{r}\right)$ are known (see Section 3). We shall rewrite the one by L. Mirsky [5] to express the correlation functions as a sum, namely

$$
=\sum_{0 \leq l^{\prime}<l^{\prime \prime} \leq r} \sum_{\substack{\mu^{2}\left(d_{l^{\prime}, l^{\prime \prime}}\right)=1 \\ d_{l^{\prime}, l^{\prime \prime}}^{2} k_{l^{\prime}}-k_{l^{\prime \prime}}}} \sum_{m_{0}, m_{1}, \ldots, m_{r} \geq 0}(-1)^{\sum_{l=0}^{r} m_{l}} \sum_{\substack{\mathcal{P}_{l} \subset \mathcal{P}, 0 \leq l \leq r \\\left|\mathcal{P}_{l}\right|=m_{l} \\\left[\mathcal{P}_{l^{\prime}} \cap \mathcal{P}_{l^{\prime \prime}}=d_{l^{\prime}, l^{\prime \prime}}\right.}} \frac{1}{\left[\bigcup_{l=0}^{r} \mathcal{P}_{l}\right]^{2}} .
$$

The above formula, although complicated, plays a role in the spectral analysis of the correlation functions. Let, for example, $r=1$. For every $d \in \mathcal{Q}$ define

$$
\sigma_{d}=\sum_{m_{0}, m_{1} \geq 0}(-1)^{m_{0}+m_{1}} \sum_{\substack{\mathcal{P}_{0}, \mathcal{P}_{1} \subset \mathcal{P} \\ \mathcal{P}_{0}\left|=m_{0},\right| \mathcal{P}_{1} \mid=m_{1} \\\left[\mathcal{P}_{0} \cap \mathcal{P}_{1}\right]=d}} \frac{1}{\left[\mathcal{P}_{0} \cup \mathcal{P}_{1}\right]^{2}}
$$

Explicit formulæ for $\sigma_{d}$ are given in Section 3. Then

$$
c_{2}(k)=\sum_{\substack{\mu^{2}(d)=1 \\ d^{2} \mid k}} \sigma_{d}
$$

and the corresponding spectral measure $v$ on $\mathbb{S}^{1}$ (i.e. satisfying $c_{2}(k)=\hat{v}(k)$ by Bochner's theorem) is pure point, given as a sum of $\delta$-functions at the points $e\left(l / d^{2}\right)$, where $d \in \mathcal{Q}$. More precisely,

$$
v=\sum_{\mu^{2}(d)=1} \sigma_{d} \sum_{l=0}^{d^{2}-1} \delta_{e\left(l / d^{2}\right)},
$$

where the convergence of the series is guaranteed by Lemma 3.1 below. The spectrum (i.e. the support of $v$ ) is the group

$$
\Lambda=\left\{e\left(l / d^{2}\right): 0 \leq l \leq d^{2}-1, \mu^{2}(d)=\mu^{2}\left(\operatorname{gcd}\left(l, d^{2}\right)\right)=1\right\} \subset \mathbb{S}^{1} .
$$

Notice that every element of $\Lambda$ is represented uniquely. Moreover, every rational number of the form $l / d^{2}$ such that $d$ is square-free, $0 \leq l \leq d^{2}-1$, and $\operatorname{gcd}\left(l, d^{2}\right)$ is also square-free can be written as

$$
\frac{l}{d^{2}}=\frac{l_{1}}{p_{1}^{2}}+\cdots+\frac{l_{r}}{p_{m}^{2}}
$$

for some $l_{1}, \ldots, l_{m}$, where $\left\{p_{1}, \ldots, p_{m}\right\}=\mathcal{P}(d)$. This representation (8) is unique if one imposes the restriction $0 \leq l_{j} \leq p_{m}^{2}-1,1 \leq j \leq m$. In other words, the group $\Lambda$ is isomorphic to the direct $\operatorname{sum} \bigoplus_{p} \mathbb{Z} / p^{2} \mathbb{Z}$ (where only finitely many coordinates are non-zero). Therefore, $\Lambda$ is the Pontryagin dual of the direct product group

$$
\mathbb{G}=\prod_{p} \mathbb{Z} / p^{2} \mathbb{Z}
$$


which is an Abelian compact group (endowed with the product topology). In other words, $\hat{\mathbb{G}} \cong \Lambda$. Each element $\mathbf{g} \in \mathbb{G}$ is identified with a sequence $\left(g_{p^{2}}\right)_{p \in \mathcal{P}}$ indexed by $\mathcal{P}$, where

$g_{p^{2}} \in \mathbb{Z} / p^{2} \mathbb{Z}:$

$$
\mathbf{g} \equiv\left(g_{4}, g_{9}, g_{25}, g_{49}, \ldots\right) .
$$

Given $\mathbf{h} \in \mathbb{G}$, denote by $\mathbb{T}_{\mathbf{h}}: \mathbb{G} \rightarrow \mathbb{G}$ the translation $\mathbb{T}_{\mathbf{h}}(\mathbf{g})=\mathbf{g}+\mathbf{h}$. Let $\mathbb{B}$ be the natural $\sigma$-algebra on $\mathbb{G}$, and let us put the uniform measure on each $\mathbb{Z} / p^{2} \mathbb{Z}$. The corresponding product measure $\mathbb{P}$ on $\mathbb{B}$ is invariant under translations, and therefore it is the Haar measure.

The ergodic properties of translations on compact Abelian groups (also known as Kronecker systems) were studied for the first time by J. von Neumann [15]. He showed that two such ergodic translations with the same spectrum are isomorphic as measurepreserving dynamical systems. This is true in general for ergodic transformations with pure point spectrum and it plays an important role in our analysis. Later, P. R. Halmos and J. von Neumann [3] proved that every ergodic dynamical system with pure point spectrum is isomorphic to a translation on a compact Abelian group. This implies, for example, that every ergodic dynamical system with pure point spectrum is isomorphic to its inverse. For an historical survey on the isomorphism problem see [8].

\subsection{A natural dynamical system}

Consider the space $X$ of all bi-infinite sequences $x=\{x(n),-\infty<n<\infty\}$ where each $x(n)$ takes value either 0 or 1 . Denote by $\mathcal{B}$ the natural $\sigma$-algebra generated by cylinder sets, and introduce the probability measure $\Pi$ defined on $\mathcal{B}$ as follows: For every $r \geq 0$ and every $-\infty<k_{0}<k_{1}<\cdots<k_{r}<\infty$,

$$
\Pi\left\{x \in X: x\left(k_{0}\right)=x\left(k_{1}\right)=\cdots=x\left(k_{r}\right)=1\right\}=c_{r+1}\left(k_{1}-k_{0}, \ldots, k_{r}-k_{0}\right),
$$

where $c_{r+1}$ is the $(r+1)$-st correlation function (4). It is clear that (10) determines the measure $\Pi$ uniquely. We call $\Pi$ the natural measure corresponding to the set of squarefree numbers.

If $T$ is the shift on $X$, i.e. $T x=x^{\prime}, x^{\prime}(n)=x(n+1)$, then it follows immediately from (10) that $\Pi$ is invariant under $T$. We can now formulate the main result of this paper:

Main Theorem. (i) The dynamical system $(X, \mathcal{B}, \Pi, T)$ is ergodic and has pure point spectrum given by $\Lambda$.

(ii) $(X, \mathcal{B}, \Pi, T)$ is isomorphic to $\left(\mathbb{G}, \mathbb{B}, \mathbb{P}, \mathbb{T}_{\mathbf{u}}\right)$, where $\mathbf{u}=(1,1, \ldots)$.

P. Sarnak [12] states that $\left(\mathbb{G}, \mathbb{B}, \mathbb{P}, \mathbb{T}_{\mathbf{u}}\right)$ is a factor of $(X, \mathcal{B}, \Pi, T)$. His methods also allow one to show that the factor map is in fact an isomorphism. Our approach is rather different and is based on a spectral analysis of the dynamical system $(X, \mathcal{B}, \Pi, T)$. The statement in the following corollary can also be found in [12].

Corollary. The dynamical system $(X, \mathcal{B}, \Pi, T)$ is not weakly mixing, and its measuretheoretic entropy is zero. 
It is worth remarking that the main focus of [12] is on the topological dynamical systems $M=\left(O_{\mu(n)}, T\right), S=\left(O_{\mu^{2}(n)}, T\right)$ given by the shifts on the orbit closures of $(\mu(n))_{n}$ and $\left(\mu^{2}(n)\right)_{n}$, respectively. The topological entropy of $S$ is positive, equal to $\left(6 / \pi^{2}\right) \log 2$. R. Peckner [6] recently constructed a measure of maximal entropy for $S$; he showed that this measure is unique, and the corresponding dynamical system is isomorphic to the direct product of $\left(\mathbb{G}, \mathbb{B}, \mathbb{P}, \mathbb{T}_{\mathbf{u}}\right)$ and a Bernoulli shift with entropy $\left(6 / \pi^{2}\right) \log 2$. In particular, the dynamical system $(X, \mathcal{B}, \Pi, T)$ that we consider is its Pinsker factor.

Our paper is organized as follows. Section 2 includes the classical computation of the density of square-free numbers and its generalization to square-free numbers avoiding finite sets of prime factors (the proof is given in Appendix A). The latter will be used for the computation of certain relevant constants. Section 3 contains various formulæ for the correlation functions, including the derivation of (6) and (4) from the formula by L. Mirsky. Section 4 includes several useful lemmata (some of which are proven in Appendix B) concerning averages and exponential sums for the correlation functions. These results are crucial for the spectral analysis of the dynamical system $(X, \mathcal{B}, \Pi, T)$. The analysis is carried out in Section 5 and yields the first part of our Main Theorem. The analysis of the spectrum for $\left(\mathbb{G}, \mathbb{B}, \mathbb{P}, \mathbb{T}_{\mathbf{u}}\right)$ is done in Section 6 , and the second part of our Main Theorem follows from it, by means of a theorem by J. von Neumann [15].

\section{The density of $\mathcal{Q}$ and some of its subsets}

Recall that $\mathbb{E}_{N}(0)=|\{n \leq N: n \in \mathcal{Q}\}| / N$. The following theorem is very classical.

Theorem 2.1.

$$
\lim _{N \rightarrow \infty} \mathbb{E}_{N}(0)=6 / \pi^{2}
$$

Proof. We can write $\mu^{2}$ as the indicator of the set of square-free numbers by imposing the condition that its argument avoids all arithmetic progressions modulo $p^{2}$ :

$$
\mu^{2}(n)=\prod_{p}\left(1-\chi_{p^{2}}(n)\right) .
$$

In the above expression $\chi_{p^{2}}(n)$ is the indicator of the arithmetic progression $\left\{p^{2} l: l \in \mathbb{Z}\right\}$. Let us open the brackets in (12):

$$
\mu^{2}(n)=1-\sum_{p} \chi_{p^{2}}(n)+\sum_{p_{1}<p_{2}} \chi_{p_{1}^{2}}(n) \chi_{p_{2}^{2}}(n)-\sum_{p_{1}<p_{2}<p_{3}} \chi_{p_{1}^{2}}(n) \chi_{p_{2}^{2}}(n) \chi_{p_{3}^{2}}(n)+\cdots
$$

We can write

$$
\begin{aligned}
\mathbb{E}_{N}(0) & =\frac{1}{N} \sum_{n \leq N} \mu^{2}(n) \\
& =1-\sum_{p} \frac{1}{p^{2}}+\sum_{p_{1}<p_{2}} \frac{1}{\left(p_{1} p_{2}\right)^{2}}-\sum_{p_{1}<p_{2}<p_{3}} \frac{1}{\left(p_{1} p_{2} p_{3}\right)^{2}}+\cdots+\varepsilon_{N} \\
& =\prod_{p}\left(1-\frac{1}{p^{2}}\right)+\varepsilon_{N}=\frac{1}{\zeta(2)}+\varepsilon_{N}=\frac{6}{\pi^{2}}+\varepsilon_{N} .
\end{aligned}
$$

Here and below $\varepsilon_{N}$ denotes a remainder that tends to zero as $N \rightarrow \infty$. 
The statement of Theorem 1 can actually be refined as follows:

\section{Theorem 2.2.}

$$
\mathbb{E}_{N}(0)=6 / \pi^{2}+\mathcal{O}\left(N^{-1 / 2}\right) \text { as } N \rightarrow \infty
$$

In other words, $\varepsilon_{N}$ in the proof of Theorem 2.1 satisfies the estimate $\left|\varepsilon_{N}\right|=\mathcal{O}\left(N^{-1 / 2}\right)$. This result is also very classical, and is a special case of Theorem 2.3 below. Let us fix a finite set $\mathcal{S} \subset \mathcal{P}$ and define the set

$$
\mathcal{Q}_{N}^{\mathcal{S}}(0)=\left\{n \leq N: \mu^{2}(n)=1, p \in \mathcal{S} \Rightarrow p \nmid n\right\}
$$

of all square-free numbers not greater than $N$ and not divisible by any of the primes $p \in \mathcal{S}$. For example, $\mathcal{Q}_{N}^{\{2\}}(0)$ is the set of odd square-free numbers not greater than $N$. Notice that when $\mathcal{S}$ is empty we get the full set of square-free numbers, i.e. $\mathcal{Q}_{N}^{\emptyset}(0)=\mathcal{Q}_{N}(0)$. In analogy with (1), let us define

$$
\mathbb{E}_{N}^{\mathcal{S}}(0)=\left|\mathcal{Q}_{N}^{\mathcal{S}}(0)\right| / N
$$

We have the following

Theorem 2.3. For every finite $\mathcal{S} \subset \mathcal{P}$ we have

$$
\mathbb{E}_{N}^{\mathcal{S}}(0)=\frac{\alpha(\mathcal{S})}{\zeta(2)}+\mathcal{O}_{\mathcal{S}}\left(N^{-1 / 2}\right) \quad \text { as } N \rightarrow \infty, \quad \text { where } \quad \alpha(\mathcal{S})=\prod_{p \in \mathcal{S}} \frac{p}{p+1}
$$

and the constant $C(\mathcal{S})$ implied by the $\mathcal{O}_{\mathcal{S}}$-notation can be taken as

$$
C(\mathcal{S})=4 \prod_{p \in \mathcal{S}} \frac{p-1}{p}+\left(\prod_{p \in \mathcal{S}} p-1\right)-\prod_{p \in \mathcal{S}}(p-1)
$$

The proof of Theorem 2.3 is presented in Appendix A; it implies the existence of the asymptotic densities

$$
\lim _{N \rightarrow \infty} \mathbb{E}_{N}^{\mathcal{S}}(0)=\alpha(\mathcal{S}) / \zeta(2)
$$

For example, the density of the set of odd square-free numbers is $4 / \pi^{2}$ (i.e. odd and even square-free numbers are in $2: 1$ proportion). Analogously, by choosing $\mathcal{S}=\{p\}$, we see that the set of square-free numbers not divisible by $p$ is " $p$ times as large" (in the sense of density) as the set of those divisible by $p$. If, for instance, we choose $\mathcal{S}=\{2,3\}$ we obtain $\alpha(\{2,3\})=1 / 2$, and we see that $50 \%$ of the square-free numbers are not divisible by either 2 or 3 . 


\section{The formulæ for the correlation functions}

L. Mirsky [5] proved that

$$
c_{r+1}\left(k_{1}, \ldots, k_{r}\right)=\prod_{p}\left(1-\frac{A_{p}^{(r+1)}\left(k_{1}, \ldots, k_{r}\right)}{p^{2}}\right),
$$

where $A_{p}^{(r+1)}\left(k_{1}, \ldots, k_{r}\right)=\left|\left\{0, k_{1}\left(\bmod p^{2}\right), \ldots, k_{r}\left(\bmod p^{2}\right)\right\}\right|$. Notice that

$$
1 \leq A_{p}^{(r+1)}\left(k_{1}, \ldots, k_{r}\right) \leq r
$$

for finitely many $p$ and $A_{p}^{(r+1)}\left(k_{1}, \ldots, k_{r}\right)=r+1$ for infinitely many $p$. For $r=1$, we have

$$
A_{p}^{(2)}(k)= \begin{cases}1, & p^{2} \mid k \\ 2, & \text { otherwise }\end{cases}
$$

This gives, for instance,

$$
c_{2}(k)=\prod_{p^{2} \mid k}\left(1-\frac{1}{p^{2}}\right) \prod_{p^{2} \nmid k}\left(1-\frac{2}{p^{2}}\right) .
$$

It will be useful for us to write $c_{2}(k)$ (and in general $c_{r+1}\left(k_{1}, \ldots, k_{r}\right)$ ) as a sum. Recall the definition of $\sigma_{d}$ from Section 1.1. We prove the following formula for $\sigma_{d}$ :

Lemma 3.1.

$$
\sigma_{d}=\frac{1}{d^{2}} \prod_{p \nmid d}\left(1-\frac{2}{p^{2}}\right) .
$$

Proof. Recall that, since $d$ is square-free, $|\mathcal{P}(d)|=\omega(d)$. By setting $m=m_{1}-\omega(d)$ and $M=m_{1}+m_{2}-2 \omega(d)$ in (5) we obtain

$$
\begin{aligned}
\sigma_{d} & =\sum_{0 \leq m \leq M}(-1)^{-2 \omega(d)}(-1)^{M}\left(\begin{array}{c}
M \\
m
\end{array}\right) \sum_{\substack{\mathcal{P}^{\prime} \subset \mathcal{P} \backslash \mathcal{P}(d) \\
\left|\mathcal{P}^{\prime}\right|=M}} \frac{1}{d^{2}} \frac{1}{\left[\mathcal{P}^{\prime}\right]^{2}} \\
& =\frac{1}{d^{2}} \sum_{M \geq 0} \sum_{\substack{\mathcal{P}^{\prime} \subset \mathcal{P} \backslash \mathcal{P}(d) \\
\left|\mathcal{P}^{\prime}\right|=M}} \frac{(-2)^{M}}{\left[\mathcal{P}^{\prime}\right]^{2}}=\frac{1}{d^{2}} \prod_{p \nmid d}\left(1-\frac{2}{p^{2}}\right) .
\end{aligned}
$$

In particular, Lemma 3.1 shows that $\sigma_{d}$ is positive and bounded away from zero and infinity. More precisely

$$
0<\sigma_{1} \leq \sigma_{d}<6 / \pi^{2}
$$

where $\sigma_{1}=\prod_{p}\left(1-2 / p^{2}\right) \approx 0.3226340989$. We can also rewrite $\sigma_{d}=\sigma_{1} \cdot \prod_{p \mid d} \frac{1}{p^{2}-2}$. 
Proposition 3.2. Let $k$ be an arbitrary integer. Then

$$
c_{2}(k)=\sum_{\substack{\mu^{2}(d)=1 \\ d^{2} \mid k}} \sigma_{d}
$$

Proof. Since $\mathcal{P}_{2}(k)=\left\{p: p^{2} \mid k\right\}$ and $\mathcal{D}(k)=\left\{\prod_{p \in \mathcal{P}^{\prime}} p: \mathcal{P}^{\prime} \subset \mathcal{P}_{2}(k)\right\}$, Lemma 3.1 gives

$$
\begin{aligned}
\sum_{d \in \mathcal{D}(k)} \sigma_{d} & =\sum_{d \in \mathcal{D}(k)} \frac{1}{d^{2}} \prod_{p \nmid d}\left(1-\frac{2}{p^{2}}\right)=\prod_{p}\left(1-\frac{2}{p^{2}}\right) \sum_{d \in \mathcal{D}(k)} \frac{1}{d^{2}} \prod_{p \mid d}\left(1-\frac{2}{p^{2}}\right)^{-1} \\
& =\prod_{p}\left(1-\frac{2}{p^{2}}\right) \sum_{d \in \mathcal{D}(k)} \prod_{p \mid d} \frac{1}{p^{2}-2}=\prod_{p}\left(1-\frac{2}{p^{2}}\right) \prod_{p \in \mathcal{P}_{2}(d)}\left(1+\frac{1}{p^{2}-2}\right) \\
& =\prod_{p^{2} \mid k}\left(1-\frac{1}{p^{2}}\right) \prod_{p^{2} \nmid k}\left(1-\frac{2}{p^{2}}\right)=c_{2}(k)
\end{aligned}
$$

by (16).

In particular, if $k=0$, then $\mathcal{D}(0)=\mathcal{Q}$ and $\mathcal{P}_{2}(0)=\mathcal{P}$ and we retrieve the known fact

$$
c_{2}(0)=\sum_{\mu^{2}(d)=1} \sigma_{d}=\prod_{p}\left(1-\frac{1}{p^{2}}\right)=\frac{6}{\pi^{2}} .
$$

Remark 3.3. Proposition 3.2 shows that the value of $c_{2}(k)$ depends on the arithmetic properties of $k$. This fact is certainly very unusual from the point of view of probability theory and statistical mechanics. If $k$ is square-free, then the function $c_{2}(k)$ takes the constant value $\sigma_{1}$. Analogously, $c_{2}(k)$ is constant along any subsequence of numbers $k$ sharing the same set of divisors that are the square of a square-free number. If we define $\mathcal{D}(k):=\left\{d: \mu^{2}(d)=1, d^{2} \mid k\right\}$, then $\mathcal{D}(k)=\mathcal{D}\left(k^{\prime}\right) \Rightarrow c_{2}(k)=c_{2}\left(k^{\prime}\right)$. The opposite implication follows from (17). Observe that every set $\mathcal{D}(k)$ is of the form

$$
\mathcal{D}(k)=\left\{\prod_{p \in \mathcal{P}^{\prime}} p: \mathcal{P}^{\prime} \subseteq \mathcal{P}_{2}(k)\right\},
$$

where $\mathcal{P}_{2}(k)=\left\{p: p^{2} \mid k\right\}$. This means that $|\mathcal{D}(k)|=2^{\left|\mathcal{P}_{2}(k)\right|}$ and $\mathcal{D}(k)=\mathcal{D}\left(k^{\prime}\right) \Leftrightarrow$ $\mathcal{P}_{2}(k)=\mathcal{P}_{2}\left(k^{\prime}\right)$. The set of $k$ such that $\mathcal{P}_{2}(k)=\emptyset$ is the set of square-free numbers, and we know that it has positive density (equal to $6 / \pi^{2}$, given by (2)). In general, we have the following

Proposition 3.4 (Density of the level sets of $c_{2}$ ). Fix a square-free number $d$. Then the density of those $k$ 's such that $c_{2}(k)=c_{2}\left(d^{2}\right)$ exists and is given by

$$
\mathfrak{d}\left(d^{2}\right):=\lim _{N \rightarrow \infty} \frac{1}{N}\left|\left\{k \leq N: c_{2}(k)=c_{2}\left(d^{2}\right)\right\}\right|=\frac{6}{\pi^{2}} \prod_{p \mid d} \frac{1}{p^{2}-1} .
$$


Proof. If $\mathcal{P}(d)=\left\{p_{1}, \ldots, p_{m}\right\}$ then $k$ satisfies $c_{2}(k)=c_{2}\left(d^{2}\right)$ if and only if it is of the form $k=p_{1}^{a_{1}} \cdots p_{m}^{a_{m}} q$, where $\mu^{2}(q)=1, a_{j} \geq 2$, and $p_{j} \nmid q$ for every $j=1, \ldots, m$. Fix $a_{1}, \ldots, a_{m} \geq 2$. Then

$$
\begin{aligned}
\frac{1}{N} \mid\left\{k \leq N: k=p_{1}^{a_{1}} \cdots p_{m}^{a_{m}} q, \mu^{2}(q)=1, p_{j}\right. & \nmid q \text { for } j=1, \ldots, m\} \mid \\
& =\frac{1}{p_{1}^{a_{1}} \cdots p_{m}^{a_{m}}} \mathbb{E}_{\left(N /\left(p_{1}^{a_{1}} \cdots p_{m}^{a_{m}}\right)\right)}^{\left\{p_{1}, \ldots, p_{m}\right\}}(0)
\end{aligned}
$$

and, by Theorem 2.3, the limit as $N \rightarrow \infty$ is

$$
\frac{6}{\pi^{2}} \frac{1}{p_{1}^{a_{1}} \cdots p_{m}^{a_{m}}} \prod_{j=1}^{m} \frac{p_{j}}{p_{j}+1}=\prod_{j=1}^{m} \frac{1}{p_{j}^{a_{j}-1}\left(p_{j}+1\right)} .
$$

Now, by summing over all $a_{j} \geq 2$, we obtain

$$
\frac{6}{\pi^{2}} \prod_{j=1}^{m} \frac{1}{p_{j}+1} \sum_{a_{j} \geq 2} \frac{1}{p_{j}^{a_{j}-1}}=\frac{6}{\pi^{2}} \prod_{j=1}^{m} \frac{1}{\left(p_{j}+1\right)\left(p_{j}-1\right)}=\frac{6}{\pi^{2}} \prod_{j=1}^{m} \frac{1}{p_{j}^{2}-1}
$$

and the proposition is proven.

Remark 3.5. The argument in the proof of Proposition 3.4 will also be used in Appendix B. We can check that

$$
\begin{aligned}
\sum_{\mu^{2}(d)=1} \mathfrak{d}\left(d^{2}\right) & =\sum_{m \geq 0} \sum_{\substack{\mathcal{P}^{\prime} \subset \mathcal{P} \\
\left|\mathcal{P}^{\prime}\right|=m}} \frac{6}{\pi^{2}} \prod_{p \in \mathcal{P}^{\prime}} \frac{1}{p^{2}-1}=\frac{6}{\pi^{2}} \prod_{p}\left(1+\frac{1}{p^{2}-1}\right) \\
& =\frac{6}{\pi^{2}} \prod_{p}\left(1-\frac{1}{p^{2}}\right)^{-1}=1 .
\end{aligned}
$$

Here we present the values of $\mathfrak{d}\left(d^{2}\right)$ for square-free numbers $d \leq 17$. The sum of the corresponding densities is $\approx 97.6 \%$ and one can check that $\sum_{d \leq 42, \mu^{2}(d)=1} \mathfrak{d}\left(d^{2}\right)>99 \%$.

\begin{tabular}{cccccccccccccc}
\hline$d$ & 1 & 2 & 3 & 5 & 6 & 7 & 10 & 11 & 13 & 14 & 15 & 17 & $\ldots$ \\
$\mathfrak{d}\left(d^{2}\right)$ & $\frac{6}{\pi^{2}}$ & $\frac{2}{\pi^{2}}$ & $\frac{3}{4 \pi^{2}}$ & $\frac{1}{4 \pi^{2}}$ & $\frac{1}{4 \pi^{2}}$ & $\frac{1}{8 \pi^{2}}$ & $\frac{1}{12 \pi^{2}}$ & $\frac{1}{20 \pi^{2}}$ & $\frac{1}{28 \pi^{2}}$ & $\frac{1}{24 \pi^{2}}$ & $\frac{1}{32 \pi^{2}}$ & $\frac{1}{48 \pi^{2}}$ & $\cdots$ \\
\hline
\end{tabular}

One can also compute the limit

$$
\lim _{N \rightarrow \infty} \frac{1}{N} \sum_{n=1}^{N} c_{2}(n)=\left(6 / \pi^{2}\right)^{2} \approx 0.3695753612
$$

by considering the series $\sum_{\mu^{2}(d)=1} \mathfrak{d}\left(d^{2}\right) c_{2}\left(d^{2}\right)$ and using Proposition 3.4 and Lemma 3.1. We shall retrieve this fact from the more general result of Lemma 4.4. Figure 1 summarizes the structure of the second correlation function. 


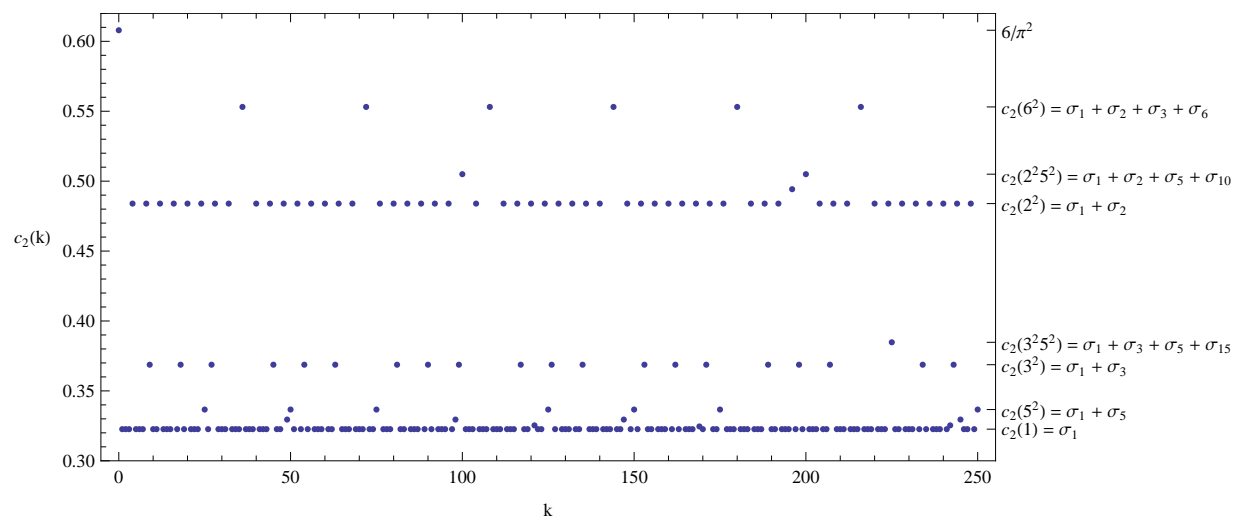

Fig. 1. The second correlation function $c_{2}(k)$ and its level sets.

Let us address the case of higher order correlation functions.

Proposition 3.6. Let $k_{1}, \ldots, k_{r}$ be such that all the differences $k_{l^{\prime}}-k_{l^{\prime \prime}}, 0 \leq l^{\prime}<l^{\prime \prime} \leq r$, are square-free. Then

$$
c_{r+1}\left(k_{1}, \ldots, k_{r}\right)=\sum_{m_{0}, m_{1}, \ldots, m_{r} \geq 0}(-1)^{\sum_{l=0}^{r} m_{l}} \sum_{\substack{\mathcal{P}_{l} \subset \mathcal{P}, 0 \leq l \leq r \\ \mathcal{P}_{l^{\prime}} \cap \mathcal{P}_{l^{\prime \prime}}=\emptyset \text { for } l^{\prime} \neq l^{\prime \prime}}} \frac{1}{\left[\bigcup_{l=0}^{r} \mathcal{P}_{l}\right]^{2}} .
$$

For general $k_{1}, \ldots, k_{r}$ we have

$$
\begin{aligned}
& c_{r+1}\left(k_{1}, \ldots, k_{r}\right)
\end{aligned}
$$

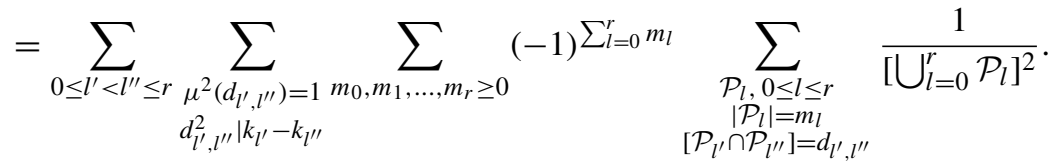

Proof. The case of $A_{p}^{(r+1)}\left(k_{1}, \ldots, k_{r}\right)=r+1$ corresponds to the case when $0, k_{1}, \ldots, k_{r}$ are distinct modulo $p^{2}$, for every prime $p$. This means that the differences $k_{l^{\prime}}-k_{l^{\prime \prime}}$ are not divisible by $p^{2}$, for every prime $p$. In other words, they are all square-free. In this case, by writing $\mathcal{P}^{\prime}=\bigcup_{l=0}^{r} \mathcal{P}_{l}$ and $m=m_{0}+\cdots+m_{r}$, the rhs of (22) equals

$$
\sum_{m \geq 0}(-1)^{m} \frac{m !}{m_{0} ! m_{1} ! \cdots m_{r} !} \sum_{\substack{\mathcal{P}^{\prime} \mathcal{P} \\|\mathcal{P}|=m}} \frac{1}{\left[\mathcal{P}^{\prime}\right]^{2}}=\prod_{p}\left(1-\frac{r+1}{p^{2}}\right) .
$$

Analogously, one can check that the quantity on the rhs of (23) equals (15) with no restrictions on $k_{1}, \ldots, k_{r}$. 
Remark 3.7. Notice that the rhs of (22) depends neither on $k_{1}, \ldots, k_{r}$ nor on the values of their differences as long as they all are square-free. Moreover, it is not enough to check that the consecutive differences $k_{1}-k_{0}, k_{2}-k_{1}, \ldots, k_{r}-k_{r-1}$ are square-free in order for all differences to be square-free. For example, if $\left(k_{1}, k_{2}, k_{3}, k_{4}\right)=(1,6,7,10)$, all consecutive differences are square-free but $2^{2} \mid k_{4}-k_{2}$ and $3^{2} \mid k_{4}-k_{1}$.

Notice also that $c_{r+1}\left(k_{1}, \ldots, k_{r}\right)$ might be zero if $r \geq 3$. For example, $c_{4}(1,2,3)=0$ since there is no $n$ such that $n, n+1, n+2, n+3$ are all square-free. All cases when $c_{r+1}\left(k_{1}, \ldots, k_{r}\right)=0$ correspond to constraints modulo $p^{2}$ for some prime $p$. This fact is clearly reflected by the general formula (15) for $c_{r+1}\left(k_{1}, \ldots, k_{r}\right)$.

Let us point out that the formula (18), (22), (23) could be derived directly by inclusion-exclusion using arithmetic progressions with step $p^{2}$. That approach-as pointed out by the anonymous referee-generates an error term that cannot be estimated elementarily. We therefore prefer the derivation of the formulæ directly from Mirsky's.

In Section 4 we shall use the following lemma by R. R. Hall [2].

Lemma 3.8. For every $0 \leq k_{1}<\cdots<k_{r}$ we have

$$
c_{r+1}\left(k_{1}, \ldots, k_{r}\right)
$$

where

$$
g(s)=\frac{6}{\pi^{2}} \mu(s) \prod_{p \mid s} \frac{1}{p^{2}-1} .
$$

Moreover, the series in (24) converges absolutely.

\subsection{Spectral analysis of $c_{2}$}

Let us expand slightly the discussion given in Section 1.1. We can rewrite (18) as

$$
c_{2}(k)=\sum_{\mu^{2}(d)=1} K_{d}(k), \quad \text { where } \quad K_{d}(k):= \begin{cases}\sigma_{d} & \text { if } d^{2} \mid k \\ 0 & \text { otherwise }\end{cases}
$$

The function $K_{d}$ is constant (equal to $\sigma_{d}$ ) along the arithmetic progression $\left\{l d^{2}: l \in \mathbb{Z}\right\}$ and 0 elsewhere. This function is the Fourier transform of a measure on the circle $\mathbb{S}^{1}$, given by a sum of $\delta$-functions at the points $e\left(l / d^{2}\right), l=0,1, \ldots, d^{2}-1$, with equal weights $\sigma_{d} / d^{2}$. A corollary of this fact is the formula (7) for the spectral measure $v$ on $\mathbb{S}^{1}$. 


\section{Averages of the correlation functions}

This section is dedicated to the proof of some results generalizing (21). For instance, one can restrict the average to those integers belonging to a certain residue class modulo a square-free $d$ (Lemmata 4.1-4.3). These averages are then used in the analysis of an exponential sums of the form $(1 / N) \sum_{n=1}^{N} \lambda^{n} c_{2}(n)$, where $\lambda$ is a complex number of modulus 1 (Lemma 4.4) in the case when $\lambda \in \Lambda$. The latter can be further extended to multiple averages of higher-order correlation functions (Lemma 4.6). These exponential sums play a crucial role in the spectral analysis of the Koopman operator for the "natural" dynamical systems $(X, \mathcal{B}, \Pi, T)$ from Section 1.2, whose invariant measure is defined by means of the correlations $c_{r+1}\left(k_{1}, \ldots, k_{r}\right)$ (see Section 5). For example, given an eigenfunction $\theta_{\lambda}: X \rightarrow \mathbb{C}$ with eigenvalue $\lambda \in \Lambda$ for the Koopman operator, we will see that its correlation with the projection onto the $s$-th coordinate $x \mapsto x(s) \in\{0,1\}$, i.e. the inner product $\left\langle x(s), \theta_{\lambda}\right\rangle_{L^{2}(X, \mathcal{B}, \Pi)}$, is given by $\lambda^{s} \lim _{N \rightarrow \infty}(1 / N) \sum_{n=1}^{N} \lambda^{n} c_{2}(n)$, and we will use the explicit form of this limit as function of $\lambda$ to study the set $\left\{\theta_{\lambda}\right\}_{\lambda \in \Lambda}$ of all eigenfunctions. The proofs of the first three lemmata are given in Appendix B.

Lemma 4.1. Let $d$ be square-free. Then

$$
\lim _{n \rightarrow \infty} \frac{1}{N} \sum_{l \leq N} c_{2}\left(d^{2} l\right)=\left(\frac{6}{\pi^{2}}\right)^{2} \prod_{p \in \mathcal{P}(d)} \frac{p^{2}}{p^{2}-1}
$$

Lemma 4.2. Let $d$ be square-free and let $1 \leq t \leq d^{2}-1, \operatorname{gcd}\left(d^{2}, t\right)=g \geq 1$, where $g$ is square-free. Then

$$
\lim _{n \rightarrow \infty} \frac{1}{N} \sum_{l \leq N} c_{2}\left(d^{2} l+t\right)=\left(\frac{6}{\pi^{2}}\right)^{2} \prod_{p \in \mathcal{P}(d)} \frac{p^{2}\left(p^{2}-2\right)}{\left(p^{2}-1\right)^{2}}
$$

Lemma 4.3. Let $d$ be square-free, and let $1 \leq t \leq d^{2}-1, \operatorname{gcd}\left(t, d^{2}\right)=g \geq 1$. Then

$$
\lim _{n \rightarrow \infty} \frac{1}{N} \sum_{l \leq N} c_{2}\left(d^{2} l+t\right)=\left(\frac{6}{\pi^{2}}\right)^{2} \prod_{p \in \mathcal{P}(d)} \frac{p^{2}\left(p^{2}-2\right)}{\left(p^{2}-1\right)^{2}} \prod_{p \in \mathcal{P}_{2}(g)} \frac{p^{2}-1}{p^{2}-2}
$$

The following two lemmata deal with exponential sums involving the second and the third correlation functions. Recall the function $g$ from (25).

Lemma 4.4. Let $\lambda=e\left(l / d^{2}\right) \in \Lambda$. Then the limit

$$
\mathfrak{Y}_{2}(\lambda)=\lim _{N \rightarrow \infty} \frac{1}{N} \sum_{n=1}^{N} \lambda^{n} c_{2}(n)
$$

exists and equals $g(d)^{2}$. 
Proof. We can write $n=d^{2} l+t$ for some $l \geq 0$ and $0 \leq t \leq d^{2}-1$ and set

$$
I_{N}(\lambda)=\frac{1}{N} \sum_{n \leq N} \lambda^{n} c_{2}(n)=\sum_{t=0}^{d^{2}-1} I_{N}^{(t)}\left(l, d^{2}\right),
$$

where

$$
I_{N}^{(t)}(\lambda)=\frac{1}{N} \sum_{m \leq(N-t) / d^{2}} e\left(\frac{l t}{d^{2}}\right) c_{2}\left(d^{2} m+t\right)
$$

Lemma 4.1 gives

$$
\lim _{N \rightarrow \infty} I_{N}^{(0)}(\lambda)=\frac{1}{d^{2}}\left(\frac{6}{\pi^{2}}\right)^{2} \prod_{p \mid d} \frac{p^{2}}{p^{2}-1} .
$$

For $t \neq 0$, the value of $\lim _{N \rightarrow \infty} I_{N}^{(t)}(\lambda)$ is given by Lemmata 4.2-4.3. It depends only on $\mathcal{P}_{2}(g)$, where $g=\operatorname{gcd}\left(t, d^{2}\right)$. More explicitly,

$$
\lim _{N \rightarrow \infty} I_{N}^{(t)}(\lambda)=e\left(\frac{l t}{d^{2}}\right) \frac{1}{d^{2}}\left(\frac{6}{\pi^{2}}\right)^{2} \prod_{p \mid d} \frac{p^{2}\left(p^{2}-2\right)}{\left(p^{2}-1\right)^{2}} \prod_{p^{2} \mid g} \frac{p^{2}-1}{p^{2}-2} .
$$

Let us introduce the notation

$$
\tau_{t}\left(d^{2}\right)=\prod_{p^{2} \mid \operatorname{gcd}\left(t, d^{2}\right)} \frac{p^{2}-1}{p^{2}-2} .
$$

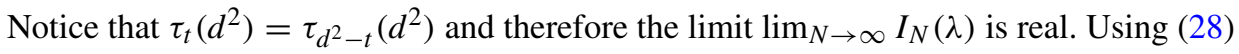
and (29) we can write

$$
\lim _{N \rightarrow \infty} I_{N}(\lambda)=\left(\frac{6}{\pi^{2}}\right)^{2} \prod_{p \mid d} \frac{1}{p^{2}-1}\left(1+\prod_{p \mid d} \frac{p^{2}-2}{p^{2}-1} \sum_{t=1}^{d^{2}-1} \cos \left(\frac{2 \pi l t}{d^{2}}\right) \tau_{t}\left(d^{2}\right)\right)
$$

and, if $\omega(d)=|\mathcal{P}(d)|=r$, then

$$
\begin{aligned}
& \sum_{t=1}^{d^{2}-1} \cos \left(\frac{2 \pi l t}{d^{2}}\right) \tau_{t}\left(d^{2}\right)=\sum_{t \leq d^{2}-1} \cos \left(\frac{2 \pi l t}{d^{2}}\right)-\sum_{p_{1} \mid d} \sum_{t \leq d^{2}-1} \cos \left(\frac{2 \pi l t}{p^{2}}\right)\left(1-\frac{p_{1}^{2}-1}{p_{1}^{2}-2}\right) \\
& +\sum_{p_{1}, p_{2} \mid d} \sum_{\substack{t \leq d^{2}-1 \\
p_{1}^{2} p_{2}^{2} \mid t}} \cos \left(\frac{2 \pi l t}{d^{2}}\right)\left(1-\frac{p_{1}^{2}-1}{p_{1}^{2}-2}\right)\left(1-\frac{p_{2}^{2}-1}{p_{2}^{2}-2}\right) \\
& -\sum_{p_{1}, p_{2}, p_{3} \mid d} \sum_{\substack{t \leq d^{2}-1 \\
p_{1}^{2} p_{2}^{2} p^{3} \mid t}} \cos \left(\frac{2 \pi l t}{d^{2}}\right)\left(1-\frac{p_{1}^{2}-1}{p_{1}^{2}-2}\right)\left(1-\frac{p_{2}^{2}-1}{p_{2}^{2}-2}\right)\left(1-\frac{p_{3}^{2}-1}{p_{3}^{2}-2}\right)+\cdots \\
& +(-1)^{r-1} \sum_{p_{1}, \ldots, p_{r-1} \mid d} \sum_{\substack{t \leq d^{2}-1 \\
p_{1}^{2} \cdots p_{r-1}^{2} \mid t}} \cos \left(\frac{2 \pi l t}{d^{2}}\right)\left(1-\frac{p_{1}^{2}-1}{p_{1}^{2}-2}\right) \cdots\left(1-\frac{p_{r-1}^{2}-1}{p_{r-1}^{2}-2}\right) .
\end{aligned}
$$


Recall that $\operatorname{gcd}\left(l, d^{2}\right)$ is square-free, and notice that for every $p_{1}, \ldots, p_{m} \mid d, m<r$,

$$
\sum_{\substack{t \leq d^{2}-1 \\ p_{1}^{2} \cdots p_{m}^{2} \mid t}} \cos \left(\frac{2 \pi l t}{d^{2}}\right)=-1 .
$$

Now (31) yields

$$
\begin{aligned}
& \sum_{t=1}^{d^{2}-1} \cos \left(\frac{2 \pi l t}{d^{2}}\right) \tau_{t}\left(d^{2}\right)=(-1)\left(1-\sum_{p_{1} \mid d} \frac{-1}{p_{1}^{2}-2}+\sum_{p_{1}, p_{2} \mid d} \frac{-1}{p_{1}^{2}-2} \frac{-1}{p_{2}^{2}-2}\right. \\
& \left.-\sum_{p_{1}, p_{2}, p_{3} \mid d} \frac{-1}{p_{1}^{2}-2} \frac{-1}{p_{2}^{2}-2} \frac{-1}{p_{3}^{2}-2}+\cdots+(-1)^{r-1} \sum_{p_{1}, \ldots, p_{r-1} \mid d} \frac{-1}{p_{1}^{2}-2} \cdots \frac{-1}{p_{r-1}^{2}-2}\right) \\
& =-\left(\prod_{p \mid d}\left(1-\frac{-1}{p^{2}-2}\right)-(-1)^{r} \prod_{p \mid d} \frac{-1}{p^{2}-2}\right)=\prod_{p \mid d} \frac{1}{p^{2}-2}-\prod_{p \mid d} \frac{p^{2}-1}{p^{2}-2},
\end{aligned}
$$

and (30) becomes

$$
\begin{aligned}
\lim _{N \rightarrow \infty} I_{N}(\lambda) & =\left(\frac{6}{\pi^{2}}\right)^{2} \prod_{p \mid d} \frac{1}{p^{2}-1}\left(1+\prod_{p \mid d} \frac{1}{p^{2}-1}-1\right)=\left(\frac{6}{\pi^{2}}\right)^{2} \prod_{p \mid d} \frac{1}{\left(p^{2}-1\right)^{2}} \\
& =g(d)^{2}
\end{aligned}
$$

Remark 4.5. Since $\frac{3}{4} p^{2} \leq p^{2}-1 \leq p^{2}$ for every $p$, we have

$$
\frac{1}{d^{2}} \leq \prod_{p \mid d} \frac{1}{p^{2}-1} \leq\left(\frac{4}{3}\right)^{\omega(d)} \frac{1}{d^{2}}
$$

Since $d$ is square-free, if we want to give an upper bound for $\omega(d)$ in terms of $d$ as $d \rightarrow \infty$, it is enough to consider the case when $d$ is the product of the first $r$ prime numbers: $d=p_{1} \cdots p_{r}$. In this case $\omega(d)=r$. It is known that $\log d=r \log r(1+o(1))$ as $r \rightarrow \infty$. This means that in general $\omega(d) \log \omega(d) \leq\left(1+\varepsilon_{1}\right) \log d$ for every $\varepsilon_{1}>0$, provided that $d \gg 1$. This implies $\omega(d) \leq \frac{\log d^{1+\varepsilon}}{W\left(\log d^{\left.1+\varepsilon_{1}\right)}\right.}$, where $W$ denotes the Lambert function, i.e. the solution of the equation $x=W(x) e^{W(x)}$. It is known that $W(x) \sim \log x$ as $x \rightarrow \infty$. Therefore $\omega(d) \leq \frac{\log d^{1+\varepsilon_{1}}}{\log \left(\log d^{1+\varepsilon_{1}}\right)^{1-\varepsilon_{2}}}$ for every $\varepsilon_{2}>0$ and thus

$$
\left(\frac{4}{3}\right)^{\omega(d)}=\mathcal{O}_{\varepsilon}\left(d^{\varepsilon}\right)
$$

for every $\varepsilon>0$ as $d \rightarrow \infty$. In other words, formulæ (28)-(29) give

$$
\lim _{N \rightarrow \infty} I_{N}^{(t)}\left(l, d^{2}\right)=\mathcal{O}_{\varepsilon}\left(\frac{1}{d^{2-\varepsilon}}\right) \quad \text { as } d \rightarrow \infty,
$$


for every $t=0,1, \ldots, d^{2}-1$. However, the cancellations coming from the different exponential factors $e^{2 \pi i t / d^{2}}$ in $I_{N}(d)$ are responsible for the higher order of smallness shown in (30):

$$
\lim _{N \rightarrow \infty} I_{N}\left(l, d^{2}\right)=\mathcal{O}\left(\frac{1}{d^{4-\varepsilon}}\right) \quad \text { as } d \rightarrow \infty .
$$

Lemma 4.6. Let $\lambda_{1}, \lambda_{2} \in \Lambda, \lambda_{1}=e\left(l_{1} / d_{1}^{2}\right), \lambda_{2}=e\left(l_{2} / d_{2}^{2}\right)$, and $\lambda=\lambda_{1} \lambda_{2}=e\left(l / d^{2}\right) \in \Lambda$. Then the 2-fold limit

$$
\mathfrak{Y}_{3}\left(\lambda_{1}, \lambda_{2}\right)=\lim _{\substack{N_{1} \rightarrow \infty \\ N_{2} \rightarrow \infty}} \frac{1}{N_{1} N_{2}} \sum_{n_{1}=1}^{N_{1}} \sum_{n_{2}=1}^{N_{2}} \lambda_{1}^{n_{2}} \lambda_{2}^{n_{2}} c_{3}\left(n_{1}, n_{2}\right)
$$

exists and equals $g\left(d_{1}\right) g\left(d_{2}\right) g(d)$.

Proof. Using Lemma 3.8 we can write

$$
\begin{aligned}
\mathfrak{Y}_{3}\left(\lambda_{1}, \lambda_{2}\right)= & \lim _{\substack{N_{1} \rightarrow \infty \\
N_{2} \rightarrow \infty}} \frac{1}{N_{1} N_{2}} \sum_{n_{1}=1}^{N_{1}} \sum_{n_{2}=1}^{N_{2}} \lambda_{1}^{n_{1}} \lambda_{2}^{n_{2}} \sum_{s_{0} \geq 1} \sum_{s_{1} \geq 1} \sum_{s_{2} \geq 1} g\left(s_{0}\right) g\left(s_{1}\right) g\left(s_{2}\right) \\
& \sum_{\substack{0 \leq t_{j} \leq s_{j}^{2}-1 \\
\mu^{2}\left(\operatorname{gcd}\left(t_{j}, s_{j}^{2}\right)\right)=1 \\
j=0,1,2 \\
t_{0} / s_{0}^{2}+t_{1} / s_{1}^{2}+t_{2} / s_{2}^{2} \in \mathbb{Z}}} e\left(\frac{t_{1}}{s_{1}^{2}} n_{1}+\frac{t_{2}}{s_{2}^{2}} n_{2}\right) .
\end{aligned}
$$

Let us bring the limit and the sums over $n_{1}, n_{2}$ in (35) inside the sum over $t_{0}, t_{1}, t_{2}$. For fixed $s_{0}, s_{1}, s_{2}$ we have

$$
\begin{aligned}
& \sum_{0 \leq t_{j} \leq s_{j}^{2}-1}\left(\lim _{N_{1} \rightarrow \infty} \frac{1}{N_{1}} \sum_{n_{1}=1}^{N_{1}} e\left(\left(\frac{t_{1}}{s_{1}^{2}}+\frac{l_{1}}{d_{1}^{2}}\right) n_{1}\right)\right)\left(\lim _{N_{2} \rightarrow \infty} \sum_{n_{2}=1}^{N_{2}} e\left(\left(\frac{t_{2}}{s_{2}^{2}} n_{2}+\frac{l_{2}}{d_{2}^{2}}\right) n_{2}\right)\right) \\
& \mu^{2}\left(\operatorname{gcd}\left(t_{j}, s_{j}^{2}\right)\right)=1 \\
& j=0,1,2 \\
& t_{0} / s_{0}^{2}+t_{1} / s_{1}^{2}+t_{2} / s_{2}^{2} \in \mathbb{Z}
\end{aligned}
$$

The two sums over $n_{1}, n_{2}$ can be written as

$$
\frac{1}{N_{j}} \sum_{n_{j}=1}^{N_{j}} e\left(\left(\frac{t_{j}}{s_{j}^{2}}+\frac{l_{j}}{d_{j}^{2}}\right) n_{j}\right)= \begin{cases}\frac{1}{N_{j}} \frac{\xi_{j}-\xi_{j}^{N+1}}{1-\xi_{j}} & \text { if } \xi_{j} \neq 1, \\ 1 & \text { if } \xi_{j}=1,\end{cases}
$$

where $\xi_{j}=e\left(t_{j} / s_{j}^{2}+l_{j} / d_{j}^{2}\right)$ and $j=1,2$. Thus, as $N \rightarrow \infty$, only the indices $t_{0}, t_{1}, t_{2}$ such that $\xi_{1}=\xi_{2}=1$ give a non-zero contribution to (35). This condition means

$$
\frac{t_{j}}{s_{j}^{2}}+\frac{l_{j}}{d_{j}^{2}}= \begin{cases}1 & \text { if } \lambda_{j} \neq 1, \\ 0 & \text { if } \lambda_{j}=1,\end{cases}
$$


for $j=1,2$. However, because of the conditions $0 \leq t_{j} \leq s_{j}^{2}-1$ and $\mu^{2}\left(\operatorname{gcd}\left(t_{j}, s_{j}^{2}\right)\right)=1$, the index

$$
t_{j}= \begin{cases}\left(s_{j}^{2} / d_{j}^{2}\right)\left(d_{j}^{2}-l_{j}\right) & \text { if } \lambda_{j} \neq 1 \\ 0 & \text { if } \lambda_{j}=1\end{cases}
$$

will be considered only when $s_{j}=d_{j}$. The value of $t_{0}$ is given consequently by

$$
\frac{t_{0}}{s_{0}^{2}}= \begin{cases}l / d^{2} & \text { if } \lambda_{1} \neq 1 \neq \lambda_{2}, \\ l_{1} / d_{1}^{2} & \text { if } \lambda_{1} \neq 1=\lambda_{2}, \\ l_{2} / d_{2}^{2} & \text { if } \lambda_{1}=1 \neq \lambda_{2} \\ 0 & \text { if } \lambda_{1}=1=\lambda_{2}\end{cases}
$$

In all cases this means $t_{0} / s_{0}^{2}=l / d^{2}$, and the condition $\mu^{2}\left(\operatorname{gcd}\left(t_{0}, s_{0}^{2}\right)\right)=1$ implies that $s_{0}=d$ and $t_{0}=l$. In other words, (35) becomes $\mathfrak{Y}_{3}\left(\lambda_{1}, \lambda_{2}\right)=g\left(d_{1}\right) g\left(d_{2}\right) g(d)$, and the lemma is proven.

Remark 4.7. Notice that if $\lambda_{1}=e\left(l_{1} / d_{1}^{2}\right), \lambda_{2}=e\left(l_{2} / d_{2}^{2}\right) \in \Lambda$ satisfy $\operatorname{gcd}\left(d_{1}, d_{2}\right)=1$, then

$$
\mathfrak{Y}_{3}\left(\lambda_{1}, \lambda_{2}\right)=\left(\frac{6}{\pi^{2}}\right)^{3}(-1)^{\omega\left(d_{1} d_{2}\right)} \prod_{p \mid d_{1} d_{2}} \frac{1}{\left(p^{2}-1\right)^{2}} .
$$

The product $\prod_{p \mid d}\left(p^{2}-1\right)$ appears in several formulæ above. Concerning this product, we have the following basic

Lemma 4.8. Let $d$ be square-free. Then

$$
\prod_{p \mid d}\left(p^{2}-1\right)=\left|\left\{1 \leq l \leq d^{2}-1: \mu^{2}\left(\operatorname{gcd}\left(l, d^{2}\right)\right)=1\right\}\right| .
$$

Proof. By standard inclusion-exclusion we can write the rhs of (36) as

$$
\begin{array}{r}
d^{2}-\sum_{p_{1} \mid d} \frac{d^{2}}{p_{1}^{2}}+\sum_{p_{1}, p_{2} \mid d} \frac{d^{2}}{\left(p_{1} p_{2}\right)^{2}}-\sum_{p_{1}, p_{2}, p_{3} \mid d} \frac{d^{2}}{\left(p_{1} p_{2} p_{3}\right)^{2}}+\cdots+(-1)^{\omega(d)} \\
=d^{2} \prod_{p \mid d}\left(1-\frac{1}{p^{2}}\right)=\prod_{p \mid d}\left(p^{2}-1\right) .
\end{array}
$$

\section{The spectrum of the shift operator $T$}

Recall the definition of the dynamical system $(X, \mathcal{B}, \Pi, T)$ given in Section 1.2. Denote by $U$ the operator on the Hilbert space $\mathcal{H}=L^{2}(X, \mathcal{B}, \Pi)$ given by

$$
(U f)(x)=f(T x) \text {. }
$$

Since $T$ is measure-preserving, the operator $U$ is unitary. The goal of this section is to prove the following 
Theorem 5.1. The spectrum of the operator $U$ is $\Lambda$.

Let us show that $\Lambda$ is contained in the spectrum of $U$. This fact is given by the following

Theorem 5.2. Let $\lambda=e\left(l / d^{2}\right) \in \Lambda$. Then there exists a function $\theta_{\lambda} \in \mathcal{H}$ such that

$$
\left(U \theta_{\lambda}\right)(x)=\lambda \theta_{\lambda}(x)
$$

for П-almost every $x \in X$.

Proof. Let $f_{0}(x)=x(0)$ and let $U_{\lambda}$ be the unitary operator on $\mathcal{H}$ defined by

$$
\left(U_{\lambda} h\right)(x)=\lambda^{-1} h(T x) .
$$

By the von Neumann Ergodic Theorem, the following limit exists in $\mathcal{H}$ :

$$
\begin{aligned}
\lim _{N \rightarrow \infty} \frac{1}{N} \sum_{n=1}^{N} U_{\lambda}^{n} f_{0}(x) & =\lim _{N \rightarrow \infty} \frac{1}{N} \sum_{n=1}^{N} \lambda^{-n} f_{0}\left(T^{n} x\right) \\
& =\lim _{N \rightarrow \infty} \frac{1}{N} \sum_{n=1}^{N} \lambda^{-n} x(n)=: \theta_{\lambda}(x) .
\end{aligned}
$$

The function $\theta_{\lambda}$ is $U_{\lambda}$-invariant, i.e. $\left(U_{\lambda} \theta_{\lambda}\right)(x)=\theta_{\lambda}(x)$ for $\Pi$-almost every $x \in X$. This implies that $\lambda^{-1} \theta_{\lambda}(T x)=\theta_{\lambda}(x)$, i.e. (38).

Denote by $x(s)$ the function $X \rightarrow\{0,1\}$ given by the projection of $x \in X$ onto its $s$-th coordinate. Introduce the subspace $H \subseteq \mathcal{H}$,

$$
H=\overline{\left\{\sum_{s} a_{s} x(s)\right\}}
$$

i.e. the closure of the set of all complex linear combinations of the $x(s)$ 's. Then $H$ is invariant under $U$, and by (39), all the eigenfunctions $\theta_{\lambda}$ belong to $H$. Let us remark that, since the operator $U$ is unitary, the eigenfunctions $\theta_{\lambda}$ are orthogonal to one another for different $\lambda$. Let us write

$$
x(s)=\sum_{\lambda \in \Lambda}\left\langle x(s), \theta_{\lambda}\right\rangle \theta_{\lambda} .
$$

Recall (25). We have the following

Theorem 5.3. Let $\lambda=e\left(l / d^{2}\right) \in \Lambda$. Then for every $s \in \mathbb{Z}$ we have

$$
\left\langle x(s), \theta_{\lambda}\right\rangle=\lambda^{s} g(d)^{2} .
$$

Proof. Let us use (39) and write

$$
\left\langle x(s), \theta_{\lambda}\right\rangle=\lim _{N \rightarrow \infty}\left\langle x(s), \frac{1}{N} \sum_{n=1}^{N} \lambda^{-n} x(n)\right\rangle=\lim _{N \rightarrow \infty} \frac{1}{N} \sum_{n=1}^{N} \lambda^{n}\langle x(s), x(n)\rangle .
$$


Notice that $\langle x(s), x(n)\rangle=c_{2}(n-s)$, where $c_{2}$ is the second correlation function given by Proposition 3.2. Equation (41) becomes

$$
\left\langle x(s), \theta_{\lambda}\right\rangle=\lim _{N \rightarrow \infty} \frac{1}{N} \sum_{n=1}^{N} \lambda^{n} c_{2}(n-s)=\lambda^{s} \lim _{N \rightarrow \infty} \frac{1}{N} \sum_{n=1}^{N} \lambda^{n} c_{2}(n)=\lambda^{s} \mathfrak{Y}_{2}(\lambda) .
$$

The needed statement follows now from Lemma 4.4.

Theorem 5.3 immediately implies the following

Corollary 5.4. All eigenfunctions $\theta_{\lambda}$ are non-zero.

Remark 5.5. The formula (39) can be written for an arbitrary measure-preserving map, but in most cases (e.g. automorphisms with continuous spectrum) it gives zero. Theorem 5.3 shows that in our case it is non-zero.

We can also compute the $L^{2}$-norm of each eigenfunction explicitly.

Theorem 5.6. Let $\lambda=e\left(l / d^{2}\right) \in \Lambda$. Then

$$
\left\|\theta_{\lambda}\right\|=|g(d)| .
$$

Proof. This is a straightforward application of Theorem 5.3:

$$
\begin{aligned}
\left\|\theta_{\lambda}\right\|^{2} & =\left\langle\theta_{\lambda}, \theta_{\lambda}\right\rangle=\left\langle\theta_{\lambda}, \lim _{N \rightarrow \infty} \frac{1}{N} \sum_{n=1}^{N} \lambda^{-n} x(n)\right\rangle=\lim _{N \rightarrow \infty} \frac{1}{N} \sum_{n=1}^{N} \lambda^{n} \overline{\left\langle x(s), \theta_{\lambda}\right\rangle} \\
& =\left(\frac{6}{\pi^{2}}\right)^{2} \prod_{p \mid d} \frac{1}{\left(p^{2}-1\right)^{2}} .
\end{aligned}
$$

Proposition 5.7. The set $\left\{\theta_{\lambda}\right\}_{\lambda \in \Lambda}$ of eigenfunctions is a basis for $H$.

Proof. Since the eigenfunctions are orthogonal it is enough to show that they span the space of all linear combinations of the $x(s)$ 's. We know that each atom $\{\lambda\}$ of the spectral measure $v$ (associated to the second correlation function via Bochner's theorem) corresponds to $\theta_{\lambda}$ in the space $H$ generated by linear forms, and these form a set of generators for $H$.

Let us define the normalized eigenfunctions: for $\lambda \in \Lambda$ set

$$
\tilde{\theta}_{\lambda}=\theta_{\lambda} /\left\|\theta_{\lambda}\right\|
$$

so that $\left\{\tilde{\theta}_{\lambda}\right\}_{\lambda \in \Lambda}$ is an orthonormal basis for $H$. Let us write

$$
x(s)=\sum_{\lambda \in \Lambda}\left\langle x(s), \tilde{\theta}_{\lambda}\right\rangle \tilde{\theta}_{\lambda}
$$


Since $\left\{\tilde{\theta}_{\lambda}\right\}_{\lambda}$ is an orthonormal basis for $H$, by Lemma 4.8 and Theorem 5.6 we have

$$
\begin{aligned}
\|x(s)\|^{2} & =\sum_{\lambda \in \Lambda}\left|\left\langle x(s), \tilde{\theta}_{\lambda}\right\rangle\right|^{2}=\left(\frac{6}{\pi^{2}}\right)^{2} \sum_{d \in \mathcal{Q}} \prod_{p \mid d} \frac{1}{p^{2}-1} \\
& =\left(\frac{6}{\pi^{2}}\right)^{2} \sum_{\mathcal{P}^{\prime} \subset \mathcal{P},\left|\mathcal{P}^{\prime}\right|<\infty} \prod_{p \in \mathcal{P}^{\prime}} \frac{1}{p^{2}-1}=\left(\frac{6}{\pi^{2}}\right)^{2} \prod_{p}\left(1+\frac{1}{p^{2}-1}\right)=\frac{6}{\pi^{2}} .
\end{aligned}
$$

The same argument allows us to estimate the size of the error term in the following approximation of $x(s)$ : for $D \geq 1$ let

$$
x_{D}(s)=\sum_{\substack{\lambda=e\left(l / d^{2}\right) \in \Lambda \\ d \leq D}}\left\langle x(s), \tilde{\theta}_{\lambda}\right\rangle \tilde{\theta}_{\lambda} .
$$

Arguing as in Remark 4.5, we have

$$
\left\|x(s)-x_{D}(s)\right\|^{2}=\sum_{\substack{\lambda=e\left(l / d^{2}\right) \in \Lambda \\ d>D}}\left|\left\langle x(s), \tilde{\theta}_{\lambda}\right\rangle\right|^{2}=\frac{6}{\pi^{2}} \sum_{d>D}|g(d)|=\mathcal{O}_{\varepsilon}\left(D^{-1+\varepsilon}\right)
$$

for every $\varepsilon>0$.

Let us consider the product of two eigenfunctions $\tilde{\theta}_{\lambda_{1}}$ and $\tilde{\theta}_{\lambda_{2}}$. We have the following

Theorem 5.8. Let $\lambda_{1}=e\left(l_{1} / d_{1}^{2}\right), \lambda_{2}=e\left(l_{2} / d_{2}^{2}\right) \in \Lambda$. Then

$$
\tilde{\theta}_{\lambda_{1}} \tilde{\theta}_{\lambda_{2}}=\epsilon \tilde{\theta}_{\lambda}
$$

where $\lambda=e\left(l / d^{2}\right)=\lambda_{1} \lambda_{2}$ and $\epsilon=\epsilon\left(\lambda_{1}, \lambda_{2}\right)=\mu\left(d_{1}\right) \mu\left(d_{2}\right) \mu(d)= \pm 1$.

Proof. It is enough to show that for every $s \in \mathbb{Z}$ we have

$$
\left\langle\tilde{\theta}_{\lambda_{1}} \tilde{\theta}_{\lambda_{2}}, x(s)\right\rangle=\left\langle\tilde{\theta}_{\lambda}, x(s)\right\rangle .
$$

Using the definition (39) we can write

$$
\theta_{\lambda_{1}} \theta_{\lambda_{2}}=\lim _{\substack{N_{1} \rightarrow \infty \\ N_{2} \rightarrow \infty}} \frac{1}{N_{1} N_{2}} \sum_{n=1}^{N_{1}} \sum_{n=2}^{N_{2}} \lambda_{1}^{-n_{1}} \lambda_{2}^{-n_{2}} x\left(n_{1}\right) x\left(n_{2}\right)
$$

and thus

$$
\left\langle\theta_{\lambda_{1}} \theta_{\lambda_{2}}, x(s)\right\rangle=\lim _{\substack{N_{1} \rightarrow \infty \\ N_{2} \rightarrow \infty}} \frac{1}{N_{1} N_{2}} \sum_{n=1}^{N_{1}} \sum_{n=2}^{N_{2}} \lambda_{1}^{-n_{1}} \lambda_{2}^{-n_{2}}\left\langle x\left(n_{1}\right) x\left(n_{2}\right), x(s)\right\rangle .
$$


Notice that $\left\langle x\left(n_{1}\right) x\left(n_{2}\right), x(s)\right\rangle=c_{3}\left(n_{1}-s, n_{2}-s\right)$. Therefore, by Lemma 4.6,

$$
\begin{aligned}
\left\langle\theta_{\lambda_{1}} \theta_{\lambda_{2}}, x(s)\right\rangle & =\lim _{\substack{N_{1} \rightarrow \infty \\
N_{2} \rightarrow \infty}} \frac{1}{N_{1} N_{2}} \sum_{n=1}^{N_{1}} \sum_{n=2}^{N_{2}} \lambda_{1}^{-n_{1}} \lambda_{2}^{-n_{2}} c_{3}\left(n_{1}-s, n_{2}-s\right) \\
& =\lambda^{-s} \lim _{\substack{N_{1} \rightarrow \infty \\
N_{2} \rightarrow \infty}} \frac{1}{N_{1} N_{2}} \sum_{n=1}^{N_{1}} \sum_{n=2}^{N_{2}} \lambda_{1}^{-n_{1}} \lambda_{2}^{-n_{2}} c_{3}\left(n_{1}, n_{2}\right) \\
& =\lambda^{-s} \mathfrak{Y}_{3}\left(\lambda_{1}^{-1}, \lambda_{2}^{-1}\right)=\lambda^{-s} g\left(d_{1}\right) g\left(d_{2}\right) g(d) .
\end{aligned}
$$

On the other hand, by Theorem 5.3,

$$
\left\langle\theta_{\lambda}, x(s)\right\rangle=e^{-\lambda} \mathfrak{Y}_{2}(\lambda)=\lambda^{-s} g(d)^{2} .
$$

Therefore

$$
\epsilon=\left\langle\tilde{\theta}_{\lambda_{1}} \tilde{\theta}_{\lambda_{2}}, x(s)\right\rangle\left\langle\tilde{\theta}_{\lambda}, x(s)\right\rangle^{-1}=\frac{g\left(d_{1}\right) g\left(d_{2}\right) g(d)}{\left|g\left(d_{1}\right)\right|\left|g\left(d_{2}\right)\right|} \frac{|g(d)|}{g(d)^{2}}=\mu\left(d_{1}\right) \mu\left(d_{2}\right) \mu(d) .
$$

By associativity of multiplication, $\epsilon\left(\lambda_{1}, \lambda_{2}\right) \epsilon\left(\lambda_{1} \lambda_{2}, \lambda_{3}\right)=\epsilon\left(\lambda_{2}, \lambda_{3}\right) \epsilon\left(\lambda_{1}, \lambda_{2} \lambda_{3}\right)$. Theorem 5.8 can be applied iteratively. It allows us to write all polynomial expressions in the eigenfunctions as linear expressions, and this is a very important fact.

We want to show that the set $\left\{\theta_{\lambda}\right\}_{\lambda \in \Lambda}$ of eigenfunctions is a basis for the whole space $\mathcal{H}$. We shall need the notion of unitary rings introduced by V. A. Rokhlin (see [10]).

Definition 5.9. A complex Hilbert space $\mathfrak{H}$ is called a unitary ring if and only if, for certain pairs of elements, a product is defined satisfying:

(I) If $f g$ is defined, then $f g=g f$.

(II) If $f g,(f g) h$ and $g h$ are defined, then $(f g) h=f(g h)$.

(III) If $f h$ and $g h$ are defined and $\alpha, \beta \in \mathbb{C}$, then $(\alpha f+\beta g) h=\alpha(f h)+\beta(g h)$.

(IV) There exists $e \in \mathfrak{H}$ such that $e f=f$ for every $f \in \mathfrak{H}$.

(V) If $f_{n} g$ are defined and $f_{n} \rightarrow f, f_{n} g \rightarrow h$, then $f g=h$.

(VI) The set $\mathfrak{M}=\{f \in \mathfrak{H}: f g$ is defined for all $g \in \mathfrak{H}\}$ is dense in $\mathfrak{H}$; moreover if $f g$ is defined, then there exist $f_{n} \in \mathfrak{M}$ such that $f_{n} \rightarrow f$ and $f_{n} g \rightarrow f g$.

(VII) For every $f \in \mathfrak{H}$, there exists $\bar{f} \in \mathfrak{H}$ such that $\langle f g, h\rangle=\langle g, \bar{f} h\rangle$ for all $f, g \in \mathfrak{M}$.

An important result by Rokhlin is that every unitary ring can be written as $\mathfrak{H}=$ $L^{2}(M, \mathcal{M}, m)$, where $(M, \mathcal{M}, m)$ is a Lebesgue space (see, e.g., V. A. Rokhlin ${ }^{1}$ [9]). In our case we have the unitary ring $\mathcal{H}=L^{2}(X, \mathcal{B}, \Pi)$ and the subspace $H$ which is a subring because of Theorem 5.8. In this representation a subring $\mathfrak{R} \subset \mathfrak{H}$ corresponds to a $\sigma$-subalgebra $\mathcal{N}$ of $\mathcal{M}$, i.e. $\mathfrak{R}=L^{2}\left(M, \mathcal{N},\left.m\right|_{\mathcal{N}}\right)$. Therefore $H$ is a subspace of $\mathcal{H}$, which is a Hilbert space corresponding to some $\sigma$-subalgebra $\mathcal{F}$ of $\mathcal{B}$. Let us show that

Proposition 5.10. Up to sets of measure zero, $\mathcal{F}=\mathcal{B}$. In other words, $H=\mathcal{H}$.

\footnotetext{
1 The notion of Lebesgue space used here allows points with positive measure, unlike the classical case discussed by Ya. G. Sinai [13] in the context of K-systems.
} 
Proof. Let us use Rokhlin's technique of measurable partitions (see [11]). According to it $\mathcal{F}$ corresponds to some measurable partition $\xi$ of $X$. If $\mathcal{F} \subsetneq \mathcal{B}$, then there exists a bounded, non-negative function $h(x)$ and a subset $A \in \mathcal{F}$ such that $\mathbb{E}\left(h \mid C_{\xi}\right) \geq \alpha$ for almost all $C_{\xi} \in A$ and some positive $\alpha$. Being a measurable function, $h$ can be approximated arbitrarily well in $L^{\infty}\left(X, \mathcal{F},\left.\Pi\right|_{\mathcal{F}}\right)$ by a function $h^{\prime}$ which is a polynomial in the $x(s)$ 's. Using (44) we can approximate $h^{\prime}$ in measure by a finite polynomial in the eigenfunctions $\theta_{\lambda}$. However, every such polynomial belongs to our Hilbert space $L^{2}\left(X, \mathcal{F},\left.\Pi\right|_{\mathcal{F}}\right)$ and it is measurable with respect to $\mathcal{F}$. Therefore the conditional expectation of $h^{\prime}$ with respect to $\xi$ is arbitrarily close to $h^{\prime}$, but such a function cannot approximate $h$ in measure. This shows that $H=\mathcal{H}$.

Propositions 5.10 and 5.7 immediately give the following

Corollary 5.11. The set $\left\{\theta_{\lambda}\right\}_{\lambda \in \Lambda}$ of eigenfunctions is a basis in the space $\mathcal{H}$.

This fact, together with Theorem 5.2 and Corollary 5.4, yields Theorem 5.1. It also implies the following

Theorem 5.12. The dynamical system $(X, \mathcal{B}, \Pi, T)$ is ergodic.

Proof. By shift-invariance of $\Pi$ we already know that the eigenspace spanned by the constants is at least one-dimensional. On the other hand, by Theorem 5.1, its dimension cannot be greater than one. This implies that the only invariant functions are constants $\Pi$-almost everywhere, and hence we have ergodicity.

Theorems 5.1 and 5.12 give part (i) of our Main Theorem.

Remark 5.13. One could also derive Corollary 5.11 in a different way and without using Rokhlin's theory of unitary rings and measurable partitions. The derivation, although explicit, is rather long. In fact, one can show that for every $-\infty<s_{1}<\cdots<s_{r}<\infty$ the product $x\left(s_{1}\right) \cdots x\left(s_{r}\right)$ belongs to the span of $\left\{\theta_{\lambda}\right\}_{\lambda \in \Lambda}$. For example, for $r=2$, by Theorems 5.3 and 5.8,

$$
\begin{aligned}
x\left(s_{1}\right) x\left(s_{2}\right) & =\left(\sum_{\lambda_{1} \in \Lambda}\left\langle x\left(s_{1}\right), \tilde{\theta}_{\lambda_{1}}\right\rangle \tilde{\theta}_{\lambda_{1}}\right)\left(\sum_{\lambda_{2} \in \Lambda}\left\langle x\left(s_{2}\right), \tilde{\theta}_{\lambda_{2}}\right\rangle \tilde{\theta}_{\lambda_{2}}\right) \\
& =\sum_{\lambda_{1}, \lambda_{2} \in \Lambda} \lambda_{1}^{s_{1}} \lambda_{2}^{s_{2}} g\left(d_{1}\right) g\left(d_{2}\right) \mu(d) \tilde{\theta}_{\lambda_{1} \lambda_{2}} \\
& =\sum_{\lambda \in \Lambda}\left(\mu(d) \sum_{\substack{\lambda_{1}, \lambda_{2} \in \Lambda \\
\lambda_{1} \lambda_{2}=\lambda}} \lambda_{1}^{s_{1}} \lambda_{2}^{s_{2}} g\left(d_{1}\right) g\left(d_{2}\right)\right) \tilde{\theta}_{\lambda},
\end{aligned}
$$

and one can prove that

$$
\sum_{\substack{\lambda_{1}, \lambda_{2} \in \Lambda \\ \lambda_{1} \lambda_{2}=\lambda}}\left|g\left(d_{1}\right) g\left(d_{2}\right)\right|=\mathcal{O}_{\varepsilon}\left(d^{-2+\varepsilon}\right)
$$


for every $\varepsilon>0$, where $\lambda=e\left(l / d^{2}\right)$. This implies that

$$
\sum_{\lambda \in \Lambda}\left|\sum_{\substack{\lambda_{1}, \lambda_{2} \in \Lambda \\ \lambda_{1} \lambda_{2}=\lambda}} \lambda_{1}^{s_{1}} \lambda_{2}^{s_{2}} g\left(d_{1}\right) g\left(d_{2}\right)\right|^{2}
$$

is finite.

\section{Spectral analysis for $\left(\mathbb{G}, \mathbb{B}, \mathbb{P}, \mathbb{T}_{\mathbf{u}}\right)$}

Recall the group $\mathbb{G}$ defined in (9). Let us consider the space $\mathbb{H}=L^{2}(\mathbb{G}, \mathbb{B}, \mathbb{P})$, and the unitary operator $\mathbb{U}$ on $\mathbb{H}$ defined by

$$
(\mathbb{U} f)(\mathbf{g})=f(\mathbf{g}+(1,1, \ldots)) .
$$

Theorem 6.1. The spectrum of $\mathbb{U}$ is $\Lambda$.

Proof. Consider the projection $\pi_{p^{2}}: \mathbb{G} \rightarrow \mathbb{Z} / p^{2} \mathbb{Z}, \pi_{p^{2}}(\mathbf{g})=g_{p^{2}}$. It is immediate to see that the function $\xi_{e\left(1 / p^{2}\right)}(\mathbf{g})=e\left(\pi_{p^{2}}(\mathbf{g}) / p^{2}\right)$ is an eigenfunction for $\mathbb{U}$ with eigenvalue $e\left(1 / p^{2}\right)$. By taking powers one can get any eigenfunction $\xi_{e\left(t / p^{2}\right)}$ with any eigenvalue $e\left(t / p^{2}\right)$ for $0 \leq t \leq p^{2}-1$. By multiplying different such eigenfunctions (with different $p$ ), one can obtain eigenfunctions $\xi_{\lambda}$ with an arbitrary eigenvalue $\lambda \in \Lambda$. Since $\Lambda$ is the character group of $\mathbb{G}$ and $\mathbb{T}_{\mathbf{u}}$ is a translation in $\mathbb{G}$, there are no other eigenvalues.

To conclude the proof of part (ii) of our Main Theorem we need the following

Theorem 6.2 (J. von Neumann, [15]). Two ergodic measure-preserving transformations with pure point spectrum are isomorphic if and only if they have the same spectrum.

Theorems 6.1 and 6.2 imply that $(X, \mathcal{B}, \Pi, T)$ and $\left(\mathbb{G}, \mathbb{B}, \mathbb{P}, \mathbb{T}_{\mathbf{u}}\right)$ are isomorphic as measure-preserving dynamical systems. This concludes the proof of our Main Theorem.

\section{Appendix A. The proof of Theorem 2.3}

The proof is based on the identity

$$
\left(\sum_{n=1}^{\infty} \frac{a(n)}{n^{s}}\right)\left(\sum_{n=1}^{\infty} \frac{b(n)}{n^{s}}\right)=\sum_{n=1}^{\infty} \frac{(a * b)(n)}{n^{s}},
$$

where $a * b$ is the Dirichlet convolution of $a$ and $b$ :

$$
(a * b)(n)=\sum_{d \mid n} a(d) b(n / d) .
$$

We shall be considering only the case of $s=2$ and bounded sequences $a(n)$ and $b(n)$, therefore there will be no question about convergence of the above series. We shall also use the classical identity

$$
\sum_{d \mid n} \mu(d)=0 \quad \text { for } n>1
$$


First, let us consider the case of square-free numbers not divisible by a single prime $p$, i.e. $\mathcal{S}=\{p\}$. In this case, we shall prove Theorem 2.3 by means of three lemmata, and then we shall explain how to generalize this approach to general finite sets $\mathcal{S}$.

Let $w_{p}(n)$ be the indicator of the integers not divisible by $p$, i.e.

$$
w_{p}(n)= \begin{cases}0 & \text { if } p \mid n \\ 1 & \text { otherwise }\end{cases}
$$

Lemma A.1.

$$
\mu^{2}(n) w_{p}(n)=\sum_{d: d^{2} \mid n} \mu(d) w_{p}(d) w_{p}(n / d) .
$$

Proof. If $p \mid n$, then $p \mid d$ or $p \mid n / d$ (possibly both) for every divisor $d$ of $n$. Thus $w_{p}(n) w_{p}(n / d)=0$ and the sum on the rhs of (49) is 0 (and obviously equals the lhs). If $p \nmid n$, then no divisor $d$ of $n$ will be divisible by $p$ and $w_{p}(d)=w_{p}(n / d)=1$. The sum in (49) then becomes $\sum_{d^{2} \mid n} \mu(d)$. If $n$ is square-free, then $d^{2}=1$ is the only perfect square that divides $n$, and the sum equals 1 (and clearly agrees with the lhs of (49)). If $n$ is not square-free let us write it as $n_{1} n_{2}^{2}$ where $n_{1}$ and $n_{2}$ are defined as follows. For every prime $p$ let us define $l=l(p, n)=\max \left\{j: p^{j} \mid n\right\}$; then set $n_{1}=\prod_{p: 2 \nmid l} p$ and $n_{2}=\prod_{p: 2 \mid l} p^{l / 2} \cdot \prod_{p: 2 \nmid l} p^{(l-1) / 2}$. Since $n_{1}$ is square-free, if $d^{2} \mid n$, then $d \mid n_{2}$. This means that the sum on the rhs of (49) becomes $\sum_{d \mid n_{2}} \mu(d)$ and equals 0 by (48) (thus matching the lhs). This concludes the proof.

\section{Lemma A.2.}

$$
\sum_{n=1}^{\infty} \frac{w_{p}(n)}{n^{2}}=\frac{p^{2}-1}{p^{2}} \zeta(2)
$$

Proof. The formula follows from the trivial computation

$$
\sum_{n=1}^{\infty} \frac{w_{p}(n)}{n^{2}}=\sum_{n=1}^{\infty} \frac{1}{n^{2}}-\sum_{n=1}^{\infty} \frac{1}{(p n)^{2}}=\left(1-\frac{1}{p^{2}}\right) \zeta(2) .
$$

Let us denote by $\left\{\delta_{1}(n)\right\}_{n \geq 1}$ the sequence equal to 1 if $n=1$ and 0 otherwise. Then we have

\section{Lemma A.3.}

$$
\left(\mu w_{p}\right) * w_{p}=\delta_{1} .
$$

Proof. For $n=1$ the statement is obvious since $d=1$ is the only divisor of $n$ and we have $\mu(1) w_{p}(1)^{2}=1=\delta_{1}(1)$. Let $n>1$. Then $\left(\left(\mu w_{p}\right) * w_{p}\right)(n)=\sum_{d \mid n} \mu(d) w_{p}(d) w_{p}(n / d)$. We can discuss the cases when $p \mid n$ and $p \nmid n$ separately, and argue as in the proof of Lemma A.1. In the first case we have $w_{p}(n) w_{p}(n / d)=0$ and the sum is 0 . In the second case $w_{p}(d)=w_{p}(n / d)=1$ and the sum becomes $\sum_{d \mid n} \mu(d)$, that is, 0 by (48). In other words, we have shown that, for $n>1$, we have $\left(\left(\mu w_{p}\right) * w_{p}\right)(n)=0=\delta_{1}(n)$, and this concludes the proof. 
Corollary A.4.

$$
\sum_{n=1}^{\infty} \frac{\mu(n) w_{p}(n)}{n^{2}}=\frac{p^{2}}{p^{2}-1} \frac{1}{\zeta(2)} .
$$

Proof. This is a straightforward application of Lemma A.2 and the formulæ (46)-(47) with $a=\mu w_{p}, b=w_{p}$, and (from Lemma A.3) $a * b=\delta_{1}$.

Proof of Theorem 2.3 when $\mathcal{S}=\{p\}$. Notice that $\left|\mathcal{Q}_{N}^{\{p\}}(0)\right|=\sum_{n \leq N} \mu^{2}(n) w_{p}(n)$. By Lemma A.1, we can write

$$
\left|\mathcal{Q}_{N}^{\{p\}}(0)\right|=\sum_{n \leq N} \mu^{2}(n) w_{p}(n)=\sum_{n \leq N} \sum_{d^{2} \mid n} \mu(d) w_{p}(d) w_{p}(n / d) .
$$

Now we want to exchange the two sums. Let us fix $d \leq \sqrt{N}$. For every $n$ of the form $n=m d^{2}$ we have $w_{p}(n / d)=w_{p}(m) w_{p}(d)$. Let $\eta_{d}^{\{p\}}(N)$ be the number of integers of the form $m d^{2}$ where $m \leq N / d^{2}$ and $p \nmid m$. Then

$$
\left|\mathcal{Q}_{N}^{\{p\}}(0)\right|=\sum_{d \leq \sqrt{N}} \eta_{d}^{\{p\}}(N) \mu(d) w_{p}(d)
$$

We can estimate the number $\eta_{d}^{\{p\}}(N)$ as follows. Let $\left\lfloor N / d^{2}\right\rfloor \equiv t(\bmod p), t \in$ $\{0,1, \ldots, p-1\}$. Then

$$
\eta_{d}^{\{p\}}(N)=\frac{\left\lfloor N / d^{2}\right\rfloor-t}{p}(p-1)+t=\frac{N}{d^{2}} \frac{p-1}{p}+q_{1}^{\{p\}}(d, N),
$$

where

$$
\left|q_{1}^{\{p\}}(d, N)\right| \leq \frac{p-1}{p}\left|\left\lfloor\frac{N}{d^{2}}\right\rfloor-\frac{N}{d^{2}}\right|+t\left(1-\frac{p-1}{p}\right) \leq 2 \frac{p-1}{p}=: C^{\prime}(\{p\}) .
$$

This gives

$$
\left|\mathcal{Q}_{N}^{\{p\}}(0)\right|=N \frac{p-1}{p} \sum_{d \leq \sqrt{N}} \frac{\mu(d) w_{p}(d)}{d^{2}}+q_{2}^{\{p\}}(N),
$$

where $\left|q_{2}^{\{p\}}(N)\right| \leq C^{\prime}(\{p\}) \sqrt{N}$. Now, Corollary A.4 yields

$$
\left|\mathcal{Q}_{N}^{\{p\}}(N)\right|=\frac{p}{p+1} \frac{1}{\zeta(2)} N+q_{2}^{\{p\}}(N)+q_{3}^{\{p\}}(N),
$$

where

$$
\begin{aligned}
\left|q_{3}^{\{p\}}(N)\right| & \leq N \frac{p-1}{p} \sum_{d>\sqrt{N}} \frac{1}{d^{2}} \leq N \frac{p-1}{p} \int_{\sqrt{N}}^{\infty} \frac{d x}{(x-1)^{2}} \\
& =\frac{p-1}{p} \frac{N}{\sqrt{N}-1} \leq C^{\prime \prime}(\{p\}) \sqrt{N}
\end{aligned}
$$


for every $N \geq 4$, where $C^{\prime \prime}(\{p\})=2(p-1) / p$. This concludes the proof, with $\alpha(\{p\})=$ $p /(p+1)$ and $C(\{p\})=C^{\prime}(\{p\})+C^{\prime \prime}(\{p\})$.

Let us now discuss how to adapt the above proof to the case of a general finite set $\mathcal{S} \subset \mathcal{P}$. The sequence $w_{p}$ has to be replaced by the indicator of the integers divisible by none of the primes in $\mathcal{S}$, i.e.

$$
w_{\mathcal{S}}(n)= \begin{cases}0 & \text { if } p \mid n \text { for some } p \in \mathcal{S} \\ 1 & \text { otherwise }\end{cases}
$$

Lemma A.1 is still valid if we replace $w_{p}$ by $w_{\mathcal{S}}$ :

Lemma A.5.

$$
\mu^{2}(n) w_{\mathcal{S}}(n)=\sum_{d: d^{2} \mid n} \mu(d) w_{\mathcal{S}}(d) w_{\mathcal{S}}(n / d)
$$

Lemma A.2 is replaced by an analogous statement given by inclusion-exclusion:

\section{Lemma A.6.}

$$
\sum_{n=1}^{\infty} \frac{w_{\mathcal{S}}(n)}{n^{2}}=a(\mathcal{S}) \zeta(2), \quad \text { where } a(\mathcal{S})=\prod_{p \in \mathcal{S}} \frac{p^{2}-1}{p^{2}}
$$

Proof. If $\mathcal{S}=\left\{p_{1}, \ldots, p_{k}\right\}$, then inclusion-exclusion gives

$a(\mathcal{S})$

$$
\begin{aligned}
& =\left(1-\sum_{i=1}^{k} \frac{1}{p_{i}^{2}}+\sum_{1 \leq i_{1}<i_{2} \leq k} \frac{1}{\left(p_{i_{1}} p_{i_{2}}\right)^{2}}-\sum_{1 \leq i_{1}<i_{2}<i_{3} \leq k} \frac{1}{\left(p_{i_{1}} p_{i_{2}} p_{i_{3}}\right)^{2}}+\cdots+\frac{(-1)^{k}}{\left(p_{1} \cdots p_{k}\right)^{2}}\right) \\
& =\prod_{i=1}^{k}\left(1-\frac{1}{p_{i}^{2}}\right) .
\end{aligned}
$$

Lemma A.3 also holds for $w_{\mathcal{S}}$ :

\section{Lemma A.7.}

$$
\left(\mu w_{\mathcal{S}}\right) * w_{\mathcal{S}}=\delta_{1}
$$

Finally, Corollary A.4 is replaced by

\section{Corollary A.8.}

$$
\sum_{n=1}^{\infty} \frac{\mu(n) w_{\mathcal{S}}(n)}{n^{2}}=\frac{1}{a(\mathcal{S}) \zeta(2)}
$$

Proof of Theorem 2.3 for general $\mathcal{S}=\left\{p_{1}, \ldots, p_{k}\right\} \subset \mathcal{P}$. Lemma A.5 gives

$$
\left|\mathcal{Q}_{N}^{\mathcal{S}}(0)\right|=\sum_{n \leq N} \sum_{d^{2} \mid n} \mu(d) w_{\mathcal{S}}(d) w_{\mathcal{S}}(n / d)
$$


Let us fix $d \leq \sqrt{N}$. For every $n$ of the form $n=m d^{2}$ we have $w_{\mathcal{S}}(n / d)=w_{\mathcal{S}}(m) w_{\mathcal{S}}(d)$. Let $\eta_{d}^{\mathcal{S}}(N)$ be the number of integers of the form $m d^{2}$ where $m \leq N / d^{2}$ and $p \nmid m$ for every $p \in \mathcal{S}$. Then

$$
\left|\mathcal{Q}_{N}^{\mathcal{S}}(0)\right|=\sum_{d \leq \sqrt{N}} \eta_{d}^{\mathcal{S}}(N) \mu(d) w_{\mathcal{S}}(d) .
$$

The set of numbers not divisible by any $p \in \mathcal{S}$ has density given by

$$
\begin{array}{r}
1-\sum_{i=1}^{k} \frac{1}{p_{i}}+\sum_{1 \leq i_{1}<i_{2} \leq k} \frac{1}{p_{i_{1}} p_{i_{2}}}-\sum_{1 \leq i_{1}<i_{2}<i_{3} \leq k} \frac{1}{p_{i_{1}} p_{i_{2}} p_{i_{3}}}+\cdots+\frac{(-1)^{k}}{p_{1} \cdots p_{k}} \\
=\prod_{i=1}^{k}\left(1-\frac{1}{p_{i}}\right)=\prod_{p \in \mathcal{S}} \frac{p-1}{p} .
\end{array}
$$

The estimate of $\eta_{d}^{\mathcal{S}}(N)$ comes from the following observation. If

$$
\left\lfloor N / d^{2}\right\rfloor \equiv t(\bmod [\mathcal{S}]) \quad \text { for } t \in\{0,1, \ldots,[\mathcal{S}]-1\},
$$

then

$$
\eta_{d}^{\mathcal{S}}(N)=\frac{\left\lfloor N / d^{2}\right\rfloor-t}{[\mathcal{S}]} \prod_{p \in \mathcal{S}}(p-1)+t=\frac{N}{d^{2}} \prod_{p \in \mathcal{S}} \frac{p-1}{p}+q_{1}^{\mathcal{S}}(d, N),
$$

where

$$
\begin{aligned}
\left|q_{1}^{\mathcal{S}}(d, N)\right| & \leq \prod_{p \in \mathcal{S}} \frac{p-1}{p}\left(\left\lfloor\frac{N}{d^{2}}\right\rfloor-\frac{N}{d^{2}}\right)+t\left(1-\prod_{p \in \mathcal{S}} \frac{p-1}{p}\right) \\
& \leq 2 \prod_{p \in \mathcal{S}} \frac{p-1}{p}+\left(\prod_{p \in \mathcal{S}} p-1\right)-\prod_{p \in \mathcal{S}}(p-1)=: C^{\prime}(\mathcal{S}) .
\end{aligned}
$$

This gives

$$
\left|\mathcal{Q}_{N}^{\mathcal{S}}(0)\right|=N \prod_{p \in \mathcal{S}} \frac{p-1}{p} \sum_{d \leq \sqrt{N}} \frac{\mu(d) w_{\mathcal{S}}(d)}{d^{2}}+q_{2}^{\mathcal{S}}(N),
$$

where $\left|q_{2}^{\mathcal{S}}(N)\right| \leq C^{\prime}(\mathcal{S}) \sqrt{N}$. Now Corollary A.8 yields

$$
\left|\mathcal{Q}_{N}^{\mathcal{S}}(0)\right|=\prod_{p \in \mathcal{S}} \frac{p}{p+1} \frac{1}{\zeta(2)} N+q_{2}^{\mathcal{S}}(N)+q_{3}^{\mathcal{S}}(N),
$$

where

$$
\left|q_{3}^{\mathcal{S}}(N)\right| \leq N \prod_{p \in \mathcal{S}} \frac{p-1}{p} \sum_{d>\sqrt{N}} \frac{1}{d^{2}} \leq C^{\prime \prime}(\mathcal{S}) \sqrt{N}
$$

and $C^{\prime \prime}(\mathcal{S})=2 \prod_{p \in \mathcal{S}}(p-1) / p$ for $N \geq 4$. This concludes the proof of the general case of the theorem, with $\alpha(\mathcal{S})=\prod_{p \in \mathcal{S}} p /(p+1)$ and $C(\mathcal{S})=C^{\prime}(\mathcal{S})+C^{\prime \prime}(\mathcal{S})$. 


\section{Appendix B. The proofs of Lemmata 4.1-4.3}

Proof of Lemma 4.1. Let us write

$$
l=\prod_{\bar{p} \in \overline{\mathcal{P}}}(\bar{p})^{a(\bar{p})} \cdot \prod_{p^{\prime} \in \mathcal{P}^{\prime}}\left(p^{\prime}\right)^{b\left(p^{\prime}\right)} \cdot q
$$

where $\overline{\mathcal{P}} \subseteq \mathcal{P}(d), a(\bar{p}) \geq 2$ for every $\bar{p} \in \overline{\mathcal{P}}, \mathcal{P}^{\prime} \subset \mathcal{P} \backslash \mathcal{P}(d),|\mathcal{P}|<\infty, b\left(p^{\prime}\right) \geq 2$ for every $\bar{p}^{\prime} \in \mathcal{P}^{\prime}, q$ is square-free and $p \nmid q$ for every $p \in \overline{\mathcal{P}} \cup \mathcal{P}^{\prime}$. It is clear that every $l \geq 1$ can be written uniquely as in (53). And the condition $l \leq N$ can be rewritten using the notation in (13) as

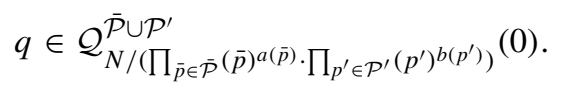

Furthermore, notice that $c_{2}\left(d^{2} l\right)$ can depend only on $d$ and $\mathcal{P}^{\prime}$ :

$$
c_{2}\left(d^{2} l\right)=\prod_{p \in P(d) \cup \mathcal{P}^{\prime}}\left(1-\frac{1}{p^{2}}\right) \prod_{p \notin \mathcal{P}(d) \cup \mathcal{P}^{\prime}}\left(1-\frac{2}{p^{2}}\right)=\sigma_{1} \prod_{p \in \mathcal{P}(d) \cup \mathcal{P}^{\prime}} \frac{p^{2}-1}{p^{2}-2} .
$$

Now we can write

$$
\begin{aligned}
& \frac{1}{N} \sum_{l \leq N} c_{2}\left(d^{2} l\right)=\sigma_{1} \prod_{p \in \mathcal{P}(d)} \frac{p^{2}-1}{p^{2}-2} \sum_{\overline{\mathcal{P}} \subseteq \mathcal{P}(d)} \sum_{\mathcal{P}} \sum_{\mathcal{P} \backslash \mathcal{P}(d)} \prod_{p^{\prime} \in \mathcal{P}^{\prime}} \frac{p^{\prime 2}-1}{p^{\prime 2}-2} \\
& \cdot \sum_{\begin{array}{c}
a(\bar{p}) \geq 2, \bar{p} \in \overline{\mathcal{P}} \\
b\left(p^{\prime}\right) \geq 2, p^{\prime} \in \mathcal{P}^{\prime}
\end{array}} \frac{1}{\prod_{\bar{p} \in \overline{\mathcal{P}}(\bar{p})^{a(\bar{p})} \cdot \prod_{p^{\prime} \in \mathcal{P}^{\prime}}\left(p^{\prime}\right)^{b\left(p^{\prime}\right)}}} \mathbb{E}_{N /\left(\prod_{\bar{p} \in \overline{\mathcal{P}}}^{\overline{\mathcal{P}} \cup(\bar{p})}\right)^{a(\bar{p})} \cdot \prod_{p^{\prime} \in \mathcal{P}^{\prime}}\left(p^{\prime}\right)^{\left.b\left(p^{\prime}\right)\right)}}(0) .
\end{aligned}
$$

Now we can use (14) while taking the limit as $N \rightarrow \infty$, and sum over all $a(\bar{p}), b\left(p^{\prime}\right) \geq 2$ as in the proof of Proposition 3.4. Notice that the sets $\mathcal{P}(d)$ and $\mathcal{P}^{\prime}$ are disjoint. We obtain

$$
\begin{aligned}
\lim _{N \rightarrow \infty} & \frac{1}{N} \sum_{l \leq N} c_{2}\left(d^{2} l\right) \\
& =\sigma_{1} \prod_{p \in \mathcal{P}(d)} \frac{p^{2}-1}{p^{2}-2} \sum_{\overline{\mathcal{P}} \subseteq \mathcal{P}(d)} \sum_{\mathcal{P}^{\prime} \subset \mathcal{P} \backslash \mathcal{P}(d)} \prod_{p^{\prime} \in \mathcal{P}^{\prime}} \frac{p^{\prime 2}-1}{p^{\prime 2}-2} \frac{6}{\pi^{2}} \prod_{p \in \overline{\mathcal{P}} \cup \mathcal{P}^{\prime}} \frac{1}{p^{2}-1} \\
& =\sigma_{1} \frac{6}{\pi^{2}} \prod_{p \in \mathcal{P}(d)} \frac{p^{2}-1}{p^{2}-2}\left(1+\frac{1}{p^{2}-1}\right) \prod_{p \in \mathcal{P} \backslash \mathcal{P}(d)}\left(1+\frac{1}{p^{2}-2}\right) \\
& =\frac{6}{\pi^{2}} \prod_{p \in \mathcal{P}(d)} \frac{p^{2}}{p^{2}-1} \prod_{p} \frac{p^{2}-1}{p^{2}}=\left(\frac{6}{\pi^{2}}\right)^{2} \prod_{p \in \mathcal{P}(d)} \frac{p^{2}}{p^{2}-1},
\end{aligned}
$$

and the lemma is proved. 
Proof of Lemma 4.2. Let us first consider the case $g=1$. Numbers of the form $\prod_{p^{\prime} \in \mathcal{P}^{\prime}} p^{\prime b\left(p^{\prime}\right)} q$, where $\mathcal{P}^{\prime} \subset \mathcal{P} \backslash \mathcal{P}(d),\left|\mathcal{P}^{\prime}\right|<\infty, b\left(p^{\prime}\right) \geq 2$ for $p^{\prime} \in \mathcal{P}^{\prime}, q$ is squarefree and $p \nmid q$ for every $p \in \mathcal{P}(d) \cup \mathcal{P}^{\prime}$ can be represented as

$$
\prod_{p^{\prime} \in \mathcal{P}^{\prime}} p^{\prime b\left(p^{\prime}\right)} \cdot q=d^{2} l+h
$$

for some $1 \leq h \leq d^{2}-1$, where $\operatorname{gcd}\left(h, d^{2}\right)=1$. Since there are $\varphi\left(d^{2}\right)$ such $h$ 's (here $\varphi$ denotes Euler's totient function) and the various $h$ 's appear with the same frequency, we have

$$
\lim _{N \rightarrow \infty} \frac{1}{N} \sum_{l \leq N} c_{2}\left(d^{2} l+t\right)=\frac{1}{\varphi\left(d^{2}\right)} \lim _{N \rightarrow \infty} \frac{1}{N} \sum_{l \leq N} \sum_{\operatorname{gcd}\left(h, d^{2}\right)=1} c_{2}\left(d^{2} l+h\right) .
$$

Notice that the condition $l \leq N$ becomes

$$
q \in \mathcal{Q}_{\left(d^{2} N+h\right) / \prod_{p^{\prime} \in \mathcal{P}^{\prime}}}^{\mathcal{P}(d) \cup \mathcal{P}^{\prime} b\left(p^{\prime}\right)}(0)
$$

and

$$
c_{2}\left(d^{2} l+t\right)=\prod_{p^{\prime} \in \mathcal{P}^{\prime}}\left(1-\frac{1}{p^{2}}\right) \prod_{p \notin \mathcal{P}^{\prime}}\left(1-\frac{2}{p^{2}}\right)=\sigma_{1} \prod_{p^{\prime} \in \mathcal{P}^{\prime}} \frac{p^{\prime 2}-1}{p^{\prime 2}-2} .
$$

Now

$$
\begin{gathered}
\frac{1}{N} \sum_{l \leq N} \sum_{\operatorname{gcd}\left(h, d^{2}\right)=1} c_{2}\left(d^{2} l+h\right)=\sigma_{1} \sum_{\mathcal{P}^{\prime} \subset \mathcal{P} \backslash \mathcal{P}(d)} \prod_{p^{\prime} \in \mathcal{P}^{\prime}} \frac{p^{\prime 2}-1}{p^{2}-2} \\
\cdot \sum_{b\left(p^{\prime}\right) \geq 2, p^{\prime} \in \mathcal{P}^{\prime}} \frac{d^{2}}{\prod_{p^{\prime} \in \mathcal{P}^{\prime}} p^{\prime b\left(p^{\prime}\right)}} \frac{d^{2} N+h}{d^{2} N} \mathbb{E}_{\left(d^{2} N+h\right) / \prod_{p^{\prime} \in \mathcal{P}^{\prime}} p^{\prime b\left(p^{\prime}\right)}}^{\mathcal{P}(d) \cup \mathcal{P}^{\prime}},
\end{gathered}
$$

and by taking the limit as $N \rightarrow \infty$ we get

$$
\begin{aligned}
& \lim _{N \rightarrow \infty} \frac{1}{N} \sum_{l \leq N} \sum_{\operatorname{gcd}\left(h, d^{2}\right)=1} c_{2}\left(d^{2} l+h\right) \\
& =\sigma_{1} \sum_{\mathcal{P}^{\prime} \subset \mathcal{P} \backslash \mathcal{P}(d)} \prod_{p^{\prime} \in \mathcal{P}^{\prime}} \frac{p^{\prime 2}-1}{p^{2}-2} \frac{6}{\pi^{2}} \prod_{p \in \mathcal{P}(d)} \frac{p^{3}}{p+1} \prod_{p^{\prime} \in \mathcal{P}^{\prime}} \frac{1}{p^{2}-1} \\
& =\sigma_{1} \frac{6}{\pi^{2}} \prod_{p \in \mathcal{P}(d)} \frac{p^{3}}{p+1} \sum_{\mathcal{P}^{\prime} \subset \mathcal{P} \backslash \mathcal{P}(d)} \prod_{p^{\prime} \in \mathcal{P}^{\prime}} \frac{1}{p^{\prime 2}-2} \\
& =\sigma_{1} \frac{6}{\pi^{2}} \prod_{p \in \mathcal{P}(d)} \frac{p^{3}}{p+1} \prod_{p \in \mathcal{P} \backslash \mathcal{P}(d)}\left(1+\frac{1}{p^{2}-2}\right) \\
& =\frac{6}{\pi^{2}} \prod_{p \in \mathcal{P}(d)} \frac{p^{3}\left(p^{2}-2\right)}{(p+1)\left(p^{2}-1\right)} \prod_{p} \frac{p^{2}-1}{p^{2}}=\left(\frac{6}{\pi^{2}}\right)^{2} \prod_{p \in \mathcal{P}(d)} \frac{p^{3}\left(p^{2}-2\right)}{(p+1)\left(p^{2}-1\right)} .
\end{aligned}
$$


Let us apply the fact that $\varphi$ is multiplicative and that $\varphi\left(p^{2}\right)=p(p-1)$. We obtain

$$
\frac{1}{\varphi\left(d^{2}\right)}=\prod_{p \in \mathcal{P}(d)} \frac{1}{p(p-1)}
$$

Now (55)-(57) yield the desired result.

Let us now consider the case when $\operatorname{gcd}\left(t, d^{2}\right)=g=\bar{p}$. In this case $d^{2}=\bar{p}^{2} d_{1}^{2}$ and $t=\bar{p} t_{1}$, where $d_{1}$ is square-free, $\bar{p} \nmid d_{1}$, and $\bar{p} \nmid t_{1}$. We can write

$$
\frac{d^{2}}{\bar{p}} l+\frac{t}{\bar{p}}=\bar{p} d_{1}^{2} l+t_{1}=\prod_{p^{\prime} \in \mathcal{P}^{\prime}} p^{\prime b\left(p^{\prime}\right)} \cdot q_{1},
$$

where $\mathcal{P}^{\prime} \subset \mathcal{P} \backslash \mathcal{P}(d),\left|\mathcal{P}^{\prime}\right|<\infty, q_{1}$ is square-free and $p \nmid q$ for every $p \in \mathcal{P}(d) \cup \mathcal{P}^{\prime}$. The condition $l \leq N$ now reads

$$
q_{1} \in \mathcal{Q}_{\left(\bar{p} d_{1}^{2} N+t_{1}\right) / \prod_{p^{\prime} \in \mathcal{P}^{\prime}}^{\mathcal{P}(d) \cup \mathcal{P}^{\prime}}}(0) .
$$

Since, by assumption, $\bar{p}^{2} \nmid d^{2} l+t$, we have

$$
c_{2}\left(d^{2} l+t\right)=c_{2}\left(\bar{p} d_{1}^{2}+t_{1}\right)=\prod_{p^{\prime} \in \mathcal{P}^{\prime}}\left(1-\frac{1}{p^{\prime 2}}\right) \prod_{p \notin \mathcal{P}^{\prime}}\left(1-\frac{2}{p^{2}}\right)=\sigma_{1} \cdot \prod_{p^{\prime} \in \mathcal{P}^{\prime}} \frac{p^{\prime 2}-1}{p^{\prime 2}-2} .
$$

Now, since $\operatorname{gcd}\left(t_{1}, \bar{p} d_{1}^{2}\right)=1$, we can use (55):

$$
\begin{aligned}
\lim _{N \rightarrow \infty} \frac{1}{N} \sum_{l \leq N} c_{2}\left(d^{2} l+t\right) & =\lim _{N \rightarrow \infty} \frac{1}{N} \sum_{l \leq N} c_{2}\left(\bar{p} d_{1}^{2} l+t_{1}\right) \\
& =\frac{1}{\varphi\left(\bar{p} d_{1}^{2}\right)} \lim _{N \rightarrow \infty} \frac{1}{N} \sum_{l \leq N} \sum_{\operatorname{gcd}\left(h_{1}, \bar{p} d_{1}^{2}\right)=1} c_{2}\left(\bar{p} d_{1}^{2}+h_{1}\right)
\end{aligned}
$$

and we can write

$$
\begin{gathered}
\frac{1}{N} \sum_{l \leq N} \sum_{\operatorname{gcd}\left(h_{1}, \bar{p} d_{1}^{2}\right)=1} c_{2}\left(\bar{p} d_{1}^{2}+h_{1}\right)=\sigma_{1} \sum_{\mathcal{P}^{\prime} \subset \mathcal{P} \backslash \mathcal{P}(d)} \prod_{p^{\prime} \in \mathcal{P}^{\prime}} \frac{p^{\prime 2}-1}{p^{2}-2} \\
\cdot \sum_{b\left(p^{\prime}\right) \geq 2, p^{\prime} \in \mathcal{P}^{\prime}} \frac{\bar{p} d_{1}^{2}}{\prod_{p^{\prime} \in \mathcal{P}^{\prime}} p^{\prime b\left(p^{\prime}\right)}} \frac{\bar{p} d_{1}^{2} N+h_{1}}{\bar{p} d_{1}^{2} N} \mathbb{E}_{\left(\bar{p} d_{1}^{2} N+h_{1}\right) / \prod_{p^{\prime} \in \mathcal{P}^{\prime}} p^{\prime b\left(p^{\prime}\right)}}(0) .
\end{gathered}
$$


1372

F. Cellarosi, Ya. G. Sinai

Notice that $\mathcal{P}(d)=\mathcal{P}\left(d_{1}\right) \cup\{\bar{p}\}$. By taking the limit as $N \rightarrow \infty$ we obtain

$$
\begin{aligned}
\lim _{N \rightarrow \infty} \frac{1}{N} \sum_{l \leq N} \sum_{\operatorname{gcd}\left(h_{1}, \bar{p} d_{1}^{2}\right)=1} c_{2}\left(\bar{p} d_{1}^{2} l+h_{1}\right) \\
=\sigma_{1} \sum_{\mathcal{P}^{\prime} \subset \mathcal{P} \backslash \mathcal{P}(d)} \prod_{p^{\prime} \in \mathcal{P}^{\prime}} \frac{p^{\prime 2}-1}{p^{\prime 2}-2} \bar{p} d_{1}^{2} \frac{6}{\pi^{2}} \prod_{p \in \mathcal{P}(d)} \frac{p}{p+1} \prod_{p^{\prime} \in \mathcal{P}^{\prime}} \frac{1}{p^{\prime 2}-1} \\
=\sigma_{1} \frac{6}{\pi^{2}} \frac{\bar{p}^{2}}{\bar{p}+1} \prod_{p \in \mathcal{P}\left(d_{1}\right)} \frac{p^{3}}{p+1} \sum_{\mathcal{P}^{\prime} \subset \mathcal{P} \backslash \mathcal{P}(d)} \prod_{p^{\prime} \in \mathcal{P}^{\prime}} \frac{1}{p^{\prime 2}-2} \\
=\sigma_{1} \frac{6}{\pi^{2}} \frac{\bar{p}^{2}}{\bar{p}+1} \prod_{p \in \mathcal{P}\left(d_{1}\right)} \frac{p^{3}}{p+1} \prod_{p \in \mathcal{P} \backslash \mathcal{P}(d)}\left(1+\frac{1}{p^{2}-2}\right) \\
=\frac{6}{\pi^{2}} \frac{\bar{p}^{2}}{\bar{p}+1} \frac{\bar{p}^{2}-2}{\bar{p}^{2}-1} \prod_{p \in \mathcal{P}\left(d_{1}\right)} \frac{p^{3}\left(p^{2}-2\right)}{(p+1)\left(p^{2}-1\right)} \prod_{p} \frac{p^{2}-1}{p^{2}} \\
=\left(\frac{6}{\pi^{2}}\right)^{2} \frac{\bar{p}^{2}\left(\bar{p}^{2}-2\right)}{(\bar{p}+1)\left(\bar{p}^{2}-1\right)} \prod_{p \in \mathcal{P}\left(d_{1}\right)} \frac{p^{3}\left(p^{2}-2\right)}{(p+1)\left(p^{2}-1\right)} .
\end{aligned}
$$

Let us use the fact that $\varphi\left(\bar{p} d_{1}^{2}\right)=\varphi(\bar{p}) \varphi\left(d_{1}^{2}\right)=(\bar{p}-1) \varphi\left(d_{1}^{2}\right)$ to obtain the formula

$$
\frac{1}{\varphi\left(\bar{p} d_{1}^{2}\right)}=\frac{1}{\bar{p}-1} \prod_{p \in \mathcal{P}\left(d_{1}\right)} \frac{1}{p(p-1)} .
$$

Now we can combine (58)-(60) to conclude the proof of the lemma. The case of a general square-free $g$ is treated analogously.

Proof of Lemma 4.3. The case when $g$ is square-free (i.e. $\mathcal{P}_{2}(g)=\emptyset$ ) is already included in Lemma 4.2. Thus, assume $\mathcal{P}_{2}(g) \neq \emptyset$. Let, for simplicity, $\operatorname{gcd}\left(t, d^{2}\right)=g=\bar{p}^{2}$ (ie. $\omega(g)=1)$, the case of $\omega(g)>1$ being analogous. We have $d^{2}=\bar{p}^{2} d_{1}^{2}$ and $t=\bar{p}^{2} t_{1}$, where $d_{1}$ is square-free and $\bar{p} \nmid d_{1}$. In particular $\operatorname{gcd}\left(t_{1}, d_{1}^{2}\right)=1$. We can write

$$
\frac{d^{2}}{\bar{p}^{2}} l+\frac{t}{\bar{p}^{2}}=d_{1}^{2} l+t_{1}=\bar{p}^{a} \prod_{p \in \mathcal{P}^{\prime}} p^{\prime b\left(p^{\prime}\right)} q_{1},
$$

where $a \geq 0, \mathcal{P}^{\prime} \subset \mathcal{P} \backslash \mathcal{P}(d),\left|\mathcal{P}^{\prime}\right|<\infty, q_{1}$ is square-free and $p \nmid q_{1}$ for every $p \in \mathcal{P}(d) \cup \mathcal{P}^{\prime}$. The condition $l \leq N$ can be written as

$$
q_{1} \in \mathcal{Q}_{\left(d_{1}^{2} N+t_{1}\right) / \prod_{p^{\prime} \in \mathcal{P}^{\prime}}}^{\left.\mathcal{P}(b) \cup \mathcal{P}^{\prime}\right)}(0),
$$

and

$$
\begin{aligned}
c_{2}\left(d^{2} l+t\right) & =c_{2}\left(\bar{p}^{2}\left(d_{1}^{2} l+t_{1}\right)\right)=\prod_{p \in \mathcal{P}^{\prime} \cup\{\bar{p}\}}\left(1-\frac{1}{p^{2}}\right) \prod_{p \notin \mathcal{P}^{\prime} \cup\{\bar{p}\}}\left(1-\frac{2}{p^{2}}\right) \\
& =\sigma_{1} \prod_{p \in \mathcal{P}^{\prime} \cup\{\bar{p}\}} \frac{p^{2}-1}{p^{2}-2} .
\end{aligned}
$$


Notice that $\mathcal{P}^{\prime}$ and $\{\bar{p}\}$ are disjoint by construction. Using (55) we see that

$$
\begin{aligned}
\lim _{N \rightarrow \infty} \frac{1}{N} \sum_{l \leq N} c_{2}\left(d^{2} l+t\right) & =\lim _{N \rightarrow \infty} \sum_{l \leq N} c_{2}\left(\bar{p}^{2}\left(d_{1}^{2} l+t_{1}\right)\right) \\
& =\frac{1}{\varphi\left(d_{1}^{2}\right)} \lim _{N \rightarrow \infty} \frac{1}{N} \sum_{l \leq N} \sum_{\operatorname{gcd}\left(h_{1}, d_{1}^{2}\right)=1} c_{2}\left(\bar{p}^{2}\left(d_{1}^{2} l+h_{1}\right)\right) .
\end{aligned}
$$

We have

$$
\begin{aligned}
\frac{1}{N} \sum_{l \leq N} \sum_{\operatorname{gcd}\left(h_{1}, d_{1}^{2}\right)=1} c_{2}\left(\bar{p}^{2}\left(d_{1}^{2} l+h_{1}\right)\right)=\sigma_{1} \sum_{\mathcal{P}^{\prime} \subset \mathcal{P} \backslash \mathcal{P}(d)} \frac{\bar{p}^{2}-1}{\bar{p}^{2}-2} \prod_{p^{\prime} \in \mathcal{P}^{\prime}} \frac{p^{\prime 2}-1}{p^{\prime 2}-2} \\
\cdot \sum_{a \geq 0} \sum_{b\left(p^{\prime}\right) \geq 2, p^{\prime} \in \mathcal{P}^{\prime}} \frac{d_{1}^{2}}{\bar{p}^{a} \prod_{p^{\prime} \in \mathcal{P}^{\prime}} p^{\prime b\left(p^{\prime}\right)}} \frac{d_{1}^{2} N+h_{1}}{d_{1}^{2} N} \mathbb{E}_{\left(d_{1}^{2} N+h_{1}\right) /\left(\bar{p}^{a} \prod_{p^{\prime} \in \mathcal{P}^{\prime}} p^{\left.\prime b\left(p^{\prime}\right)\right)}(0),\right.}^{\mathcal{P}(d) \mathcal{P}^{\prime}}
\end{aligned}
$$

and by taking the limit as $N \rightarrow \infty$ we get

$$
\begin{aligned}
\lim _{N \rightarrow \infty} & \frac{1}{N} \sum_{l \leq N} \sum_{\operatorname{gcd}\left(h_{1}, d_{1}^{2}\right)=1} c_{2}\left(\bar{p}^{2}\left(d_{1}^{2} l+h_{1}\right)\right) \\
= & \sigma_{1} \frac{\bar{p}^{2}-1}{\bar{p}^{2}-2} \sum_{\mathcal{P}^{\prime} \subset \mathcal{P} \backslash \mathcal{P}(d)} \prod_{p^{\prime} \in \mathcal{P}^{\prime}} \frac{p^{\prime 2}-1}{p^{\prime 2}-2} \frac{d_{1}^{2} \bar{p}}{\bar{p}-1} \frac{6}{\pi^{2}} \prod_{p \in \mathcal{P}(d)} \frac{p}{p+1} \prod_{p^{\prime} \in \mathcal{P}^{\prime}} \frac{1}{p^{\prime 2}-1} \\
= & \sigma_{1} \frac{6}{\pi^{2}} \frac{\bar{p}+1}{\bar{p}\left(\bar{p}^{2}-2\right)} \prod_{p \in \mathcal{P}(d)} \frac{p^{3}}{p+1} \sum_{\mathcal{P}^{\prime} \subset \mathcal{P} \backslash \mathcal{P}(d)} \prod_{p^{\prime} \in \mathcal{P}^{\prime}} \frac{1}{p^{\prime 2}-2} \\
= & \sigma_{1} \frac{6}{\pi^{2}} \frac{\bar{p}+1}{\bar{p}\left(\bar{p}^{2}-2\right)} \prod_{p \in \mathcal{P}(d)} \frac{p^{3}}{p+1} \prod_{p \in \mathcal{P} \backslash \mathcal{P}(d)}\left(1+\frac{1}{p^{2}-2}\right) \\
= & \frac{6}{\pi^{2}} \frac{\bar{p}^{2}}{\bar{p}^{2}-1} \prod_{p \in \mathcal{P}\left(d_{1}\right)} \frac{p^{3}}{p+1} \frac{p^{2}-2}{p^{2}-1} \prod_{p} \frac{p^{2}-1}{p^{2}} \\
= & \left(\frac{6}{\pi^{2}}\right)^{2} \frac{\bar{p}^{2}}{\bar{p}^{2}-1} \prod_{p \in \mathcal{P}\left(d_{1}\right)} \frac{p^{3}\left(p^{2}-2\right)}{(p+1)\left(p^{2}-1\right)} .
\end{aligned}
$$

We use again the fact that

$$
\frac{1}{\varphi\left(d_{1}^{2}\right)}=\prod_{p \in \mathcal{P}\left(d_{1}\right)} \frac{1}{p(p-1)}
$$

and combining (61)-(63), we obtain the lemma.

Acknowledgments. The authors thank M. Boyle, M. Degli Esposti, G. Forni, P. Sarnak, I. Shkredov, and I. Vinogradov for useful discussions, and the anonymous referees for their suggestions to improve on the first version of this paper. The first author's work is supported by the National Science Foundation under agreement No. DMS-0635607. The second author acknowledges the financial support from the NSF, grant No. DMS-0901235. 
Note added in proof. The authors have become aware of some independent results by M. Baake, R. V. Moody and P. A. B. Pleasants [1], where the second correlation function for square-free integers has also been computed. Moreover, a recent paper by Pleasants and C. Huck [7] addresses the problem of $m$-free points $(m \geq 2)$ in an arbitrary lattice, with a geometric notion of $m$-freeness that agrees with the one we consider when the lattice is $\mathbb{Z}$; it also includes the computation of measure-theoretical entropy for these points.

\section{References}

[1] Baake, M., Moody, R. V., Pleasants, P. A. B.: Diffraction from visible lattice points and $k$ th power free integers. Discrete Math. 221, 3-42 (2000) Zbl 1079.52013 MR 1778906

[2] Hall, R. R.: The distribution of squarefree numbers. J. Reine Angew. Math. 394, 107-117 (1989) Zbl 0653.10040 MR 0977436

[3] Halmos, P. R., von Neumann, J.: Operator methods in classical mechanics. II. Ann. of Math. (2) 43, 332-350 (1942) Zbl 0063.01888 MR 0006617

[4] Heath-Brown, D. R.: The square sieve and consecutive square-free numbers. Math. Ann. 266, 251-259 (1984) Zbl 0514.10038 MR 0730168

[5] Mirsky, L.: Arithmetical pattern problems relating to divisibility by $r$ th powers. Proc. London Math. Soc. (2) 50, 497-508 (1949) Zbl 0031.25209 MR 0028334

[6] Peckner, R.: Uniqueness of the measure of maximal entropy for the squarefree flow. Preprint

[7] Pleasants, P. A. B., Huck, C.: Entropy and diffraction of the $k$-free points in the $n$-dimensional lattice. To appear

[8] Rédei, M., Werndl, C.: On the history of the isomorphism problem of dynamical systems with special regard to von Neumann's contribution. Arch. Hist. Exact Sci. 66, 71-93 (2012) Zbl 1244.01014 MR 2855387

[9] Rokhlin, V. A.: On the problem of the classification of automorphisms of Lebesgue spaces. Dokl. Akad. Nauk SSSR 58, 189-191 (1947) (in Russian) Z Zbl 0030.24401 MR 0022591

[10] Rokhlin, V. A.: Unitary rings. Dokl. Akad. Nauk SSSR 59, 643-646 (1948) (in Russian) Zbl 0030.39801 MR 0024057

[11] Rokhlin, V. A.: On the fundamental ideas of measure theory. Mat. Sbornik 25 (67), 107-150 (1949) (in Russian) Z Zbl 0033.16904 MR 0030584

[12] Sarnak, P.: Three lectures on the Möbius function randomness and dynamics (Lecture 1). http://publications.ias.edu/sites/default/files/MobiusFunctionsLectures(2).pdf

[13] Sinai, Ya. G.: Topics in Ergodic Theory. Princeton Math. Ser. 44, Princeton Univ. Press, Princeton, NJ (1994) Zbl 0805.58005 MR 1258087

[14] Tsang, K. M.: The distribution of $r$-tuples of squarefree numbers. Mathematika 32, 265-275 (1986) (1985) Zbl 0583.10026 MR 0834495

[15] von Neumann, J.: Zur Operatorenmethode in der klassischen Mechanik. Ann. of Math. (2) 33, 587-642 (1932) JFM 58.1270.04 MR 1503078 